\title{
GLAD!
}

Revue sur le langage, le genre, les sexualités

11 | 2021

Archives, genre, sexualités, discours

\section{« Notre histoire compte »: Transmettre l'histoire des mouvements féministes et lesbiens à Genève}

"Our story matters ». Transmitting the History of Feminist and Lesbian

Movements in Geneva.

\section{Carolina Topini et Isabelle Salem Diego Sentis}

\section{OpenEdition}

\section{Journals}

Édition électronique

URL : https://journals.openedition.org/glad/3597

ISSN : 2551-0819

Éditeur

Association GSL

Référence électronique

Carolina Topini et Isabelle Salem Diego Sentis, « « Notre histoire compte » : Transmettre l'histoire des mouvements féministes et lesbiens à Genève », GLAD! [En ligne], 11 | 2021, mis en ligne le 08 janvier 2022, consulté le 29 janvier 2022. URL : http://journals.openedition.org/glad/3597

Ce document a été généré automatiquement le 29 janvier 2022.

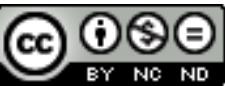

La revue GLAD! est mise à disposition selon les termes de la Licence Creative Commons Attribution Pas d'Utilisation Commerciale - Pas de Modification 4.0 International. 


\title{
« Notre histoire compte » : Transmettre l'histoire des mouvements féministes et lesbiens à Genève
}

\author{
"Our story matters ». Transmitting the History of Feminist and Lesbian \\ Movements in Geneva.
}

Carolina Topini et Isabelle Salem Diego Sentis

\section{Introduction}

Les Archives, c'est se donner un pouvoir. Nous ne devons pas nous laisser arrêter par les limites qu'on nous impose. Nous devons imaginer. Et je pense que

l'une des choses qui nous est donnée en tant que lesbiennes c'est une somme énorme d'imagination et de

force. ${ }^{1}$

Joan Nestle, fondatrice des Lesbian Herstory Archives (L.H.A)

À partir des années 1970-1980, la prise de conscience qu' " archiver c'est exister " a permis à de nombreux mouvements féministes et LGBTIQ+ de se mobiliser pour conserver et faire vivre leurs archives. "Faire archive» est une lutte en soi, une stratégie puissante et indispensable à la construction d'une mémoire collective, communautaire et vivante. Elle permet de recouvrer des voix réduites au silence et de faire circuler des imaginaires de justice et liberté. Comme le rappelle Antoine Idier, l'acte politique d'archiver constitue une réponse subversive à l'effacement: " se saisir des archives, c'est refuser de laisser aux autres le privilège épistémologique d'écrire l'histoire, c'est se dresser contre une dépossession » $(2018: 10)$. 
Dans cette contribution nous présentons le projet de collecte et transmission d'archives "Nos lieux, nos fêtes, nos combats : notre histoire compte», initié en 2018 par les associations Lestime ${ }^{2}$ et Queer Code ${ }^{3}$, et aujourd'hui mené par le collectif interassociatif Notre histoire compte. Le projet s'intéresse aux histoires des lieux de sociabilité et de militance féministes et lesbiens à Genève des années 1970 à nos jours, dans un souci de transmission transgénérationnelle des mémoires militantes, dramatiquement soumises au risque de disparition. Le projet est soutenu par la Ville de Genève dans le cadre de son projet « Mémoires LGBTIQ+ ».

Dans un premier temps, nous allons présenter les objectifs et le cadre du projet, illustrant la manière dont nous avons construit sa démarche participative et quels sont les enjeux politiques d'une telle démarche. Dans un deuxième temps, nous proposons une balade virtuelle pour découvrir une sélection d'archives en lien avec quelques moments marquants de l'histoire des mouvements féministes et lesbiens genevois. En cliquant sur les liens proposés, il sera possible d'explorer cette histoire via des ressources audiovisuelles, visuelles et sonores diffusées par les dispositifs numériques que le collectif Notre histoire compte a mis en place.

\section{« Notre histoire compte »: genèse et objectifs du projet}

À l'occasion de la commémoration des 50 ans des révoltes de Stonewall, les affrontements entre personnes LGBTIQ+ et la police de New York considérés comme le moment fondateur des marches des fiertés à travers le monde, Isabelle S.D Sentis et le Collectif Queer Code ont proposé à Lestime une coopération afin de rendre visible les mobilisations lesbiennes dans le contexte genevois, proposition qui est ensuite devenue « Notre Histoire compte ».

Très peu documentées et encore largement invisibles, les histoires des lieux de sociabilité et de militance lesbiens (bars, cafés, restaurants, centres des femmes, etc.) ont rarement fait l'objet de recherches approfondies. Leur histoire reste encore à écrire. Cependant, comme les mettent en lumière les quelques travaux qui existent, les lieux de sociabilité ont joué un rôle majeur dans la construction politique des communautés lesbiennes. Ils ne sont pas seulement des espaces de rencontre, mais également des espaces de survie et de résistance, de débats et d'échanges intellectuels, et des tentatives de construire des économies alternatives. Par ailleurs, ces espaces rendent poreuses les frontières entre l'âme plus festive et celle plus politique du mouvement (Davis, Lapovsky Kennedy 1986; Hogan 2016; Enke 2007; Chamberland 1993). C'est dans l'envie de combler ces lacunes que se situe notre projet. Des «Bals des chattes sauvages » au Centre femmes Natalie Barney, en passant par La Bretelle, chez Lili, le Brigitte, le Barbie... chaque génération a eu à Genève ses endroits de militance et de fête. Partager l'histoire des fêtes, des bars et des lieux militants d'hier et d'aujourd'hui nous a semblé une démarche stratégique pour tenter de contrer la gentrification des villes et des imaginaires (Sentis 2019), question traitée par Sarah Schulman dans son livre La Gentrification des esprits (2018).

Depuis son essor, le projet a vu le déploiement d'une dynamique intergénérationnelle très significative. La perspective historique considérée, qui s'étend des années 1970 à nos jours, a contribué à instaurer cette dynamique. Nous avons veillé à ne pas reproduire des clivages générationnels au sein du groupe, créant en revanche le sens 
d'une appartenance collective à une généalogie féministe et lesbienne aux contours mouvants. Pour cette raison, nous n'avons pas voulu fixer des bornes chronologiques rigides ni adhérer à une périodisation préétablie typique d'une certaine historiographie féministe. Sans effacer les spécificités de chaque conjoncture historique et de chaque génération politique, nous avons choisi de mettre l'accent sur l'héritage et sur les continuités plutôt que sur les discontinuités et les ruptures, trouvant des résonances inattendues dans les archives d'époques, générations et lieux très différents. L'importance de conquérir des espaces "à soi ", de se doter de moyens d'expression propres, de prendre la rue, de manifester, de faire la fête, de tisser des solidarités et des alliances au-delà des frontières géographiques, reste au fil du temps les moteurs principaux du militantisme lesbien. Au sein de ce projet, nous avons donc mobilisé les archives comme dispositif de solidarité politique et comme «site d'alliances intergénérationnelles », pour reprendre l'expression de la chercheuse Kate Eichhorn proposée dans son livre The Archival Turn in Feminism (2013).

Tout au long du projet, nous sommes restées conscientes du fait que même ce qu'on qualifie d'archives "minoritaires» (dans ce cas, les archives de l'histoire lesbienne) reste l'enjeu de rapports de pouvoir spécifiques: à leur tour, elles peuvent écarter, réduire au silence et effacer; et que seulement certain-e-x-s d'entre nous avaient le privilège de se sentir immédiatement « chez soi » dans les archives. Cette interrogation, "comment les mémoires lesbiennes sont tout aussi hantées par l'absence?", reste pour nous une question ouverte.

Le projet accorde une place importante à l'usage des technologies numériques, qui offrent de nouveaux outils pour la transmission et le partage des mémoires militantes, démultipliant les usages possibles des archives. L'objectif concret du projet était la création d'une cartographie numérique et physique des lieux genevois de fête et de militance fréquentés dans le passé et aujourd'hui par des femmes lesbiennes et bisexuelles, ainsi que par leurs amies hétérosexuelles, cis et trans*, et personnes non binaires. La mise en place de cette cartographie a soulevé de nombreuses questions, notamment sur comment visibiliser et représenter les violences subies dans l'espace urbain (et dans d'autres types d'espaces) ainsi que les violences intra-communautaires. Plus récemment, à l'occasion du Festival des visibilités lesbiennes organisé par Lestime les 24-25 avril 2021, nous avons créé une frise chronologique des mobilisations lesbiennes à Genève, incluant celles communes à la communauté gay et celles en solidarité avec la communauté trans*. 
Préparation collective de la cartographie à Lestime pendant un atelier (1) @ Isabelle S.D Sentis

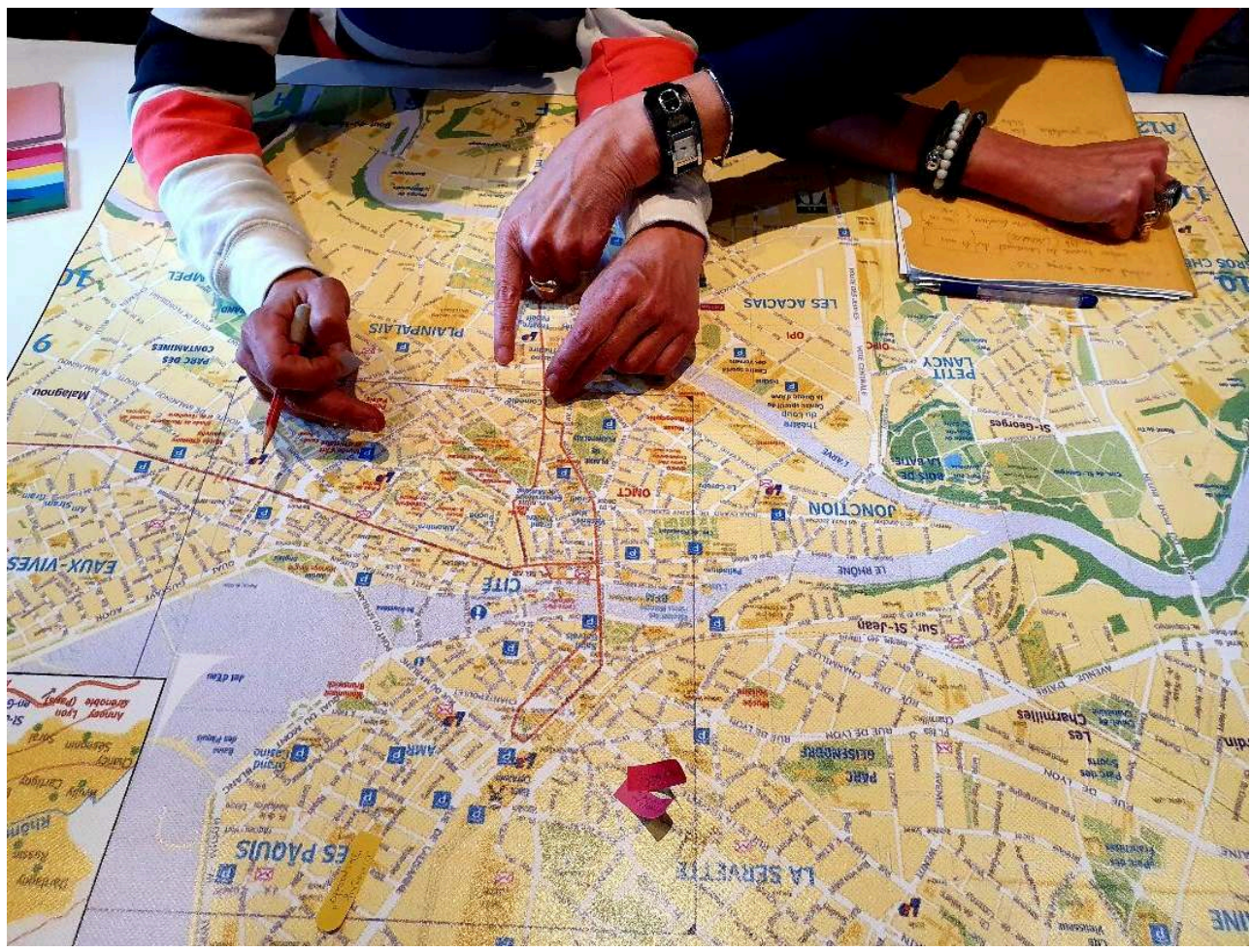

Préparation collective de la cartographie à Lestime pendant un atelier (2) @ Isabelle S.D Sentis

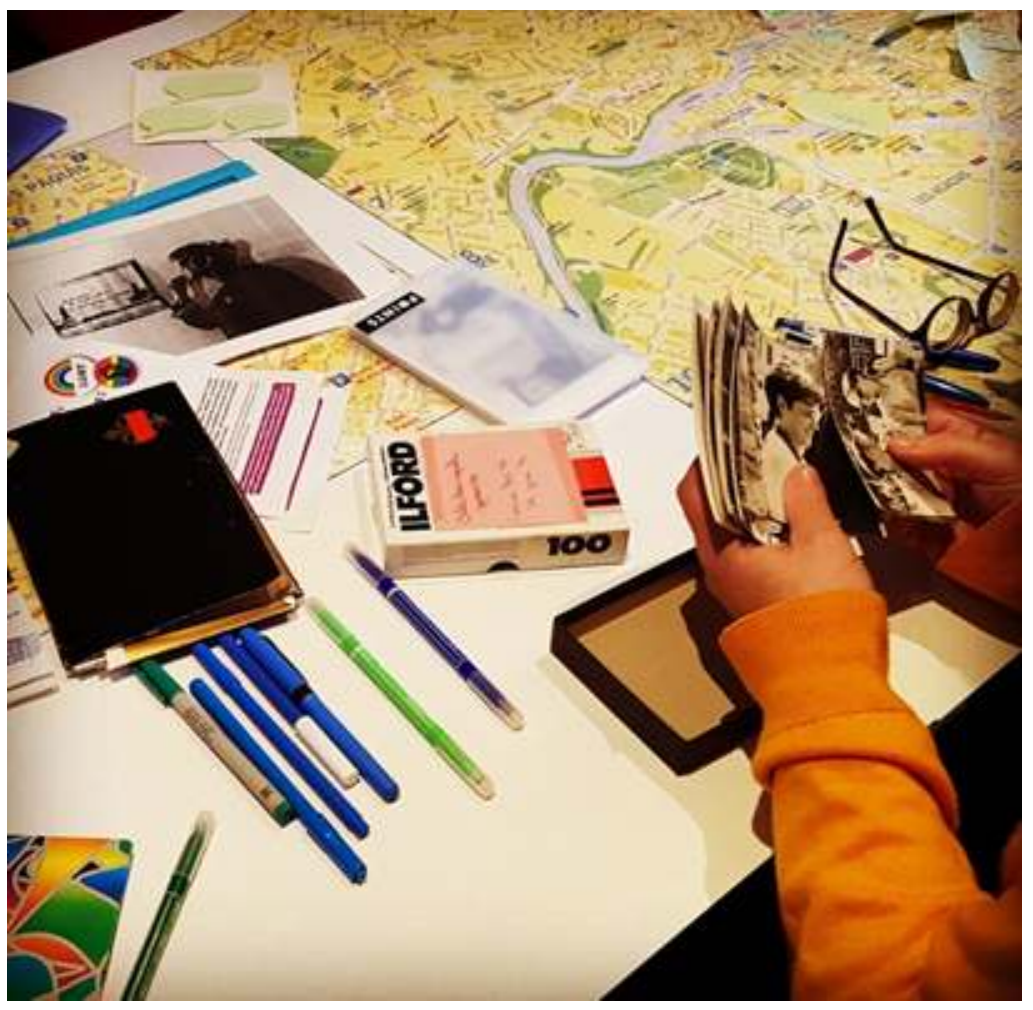




\section{Une démarche participative de renversement de l'expertise}

Notre projet s'est articulé en lien avec un contexte de réflexions militantes menées en Suisse, en France, en Belgique, aux Pays-Bas, aux États-Unis et au Canada sur le collectage, la conservation et la médiation des archives LGBTQI+. Apprendre à documenter nous-mêmes nos histoires, dans une démarche de renversement de l'expertise qui place les minorités en position d'expertes de leurs propres vies, nous a permis d'affirmer que nous étions les actrices de cette histoire et de sa conservation. Notre démarche participe à la dynamique de «l'archive vivante $»^{4}:$ il s'agit de promouvoir un rapport renouvelé à l'archive, moins élitiste et plus inclusif, redonnant aux communautés directement concernées un rôle central dans l'identification, la gestion et la transmission de leur héritage culturel et politique. Cette démarche se nourrit des expériences et des bonnes pratiques identifiées par les collectifs et centres d'archives LGBTQI existants.

Un premier atelier de redécouverte d'archives animé par Isabelle S.D Sentis, avait été proposé par Lestime en 2017 à l'occasion des 15 ans de l'association. À partir d'archives et d'images, les participantes ont réalisé collectivement un calendrier perpétuel illustré à la façon de Clit 007. Concentré Lesbien Irrésistiblement Toxique, revue/fanzine lesbienne publiée à Genève entre le 1980 et le 1986.

Atelier Calendrier perpétuel lesbien, 27-28 janvier 2017, dans les locaux de Lestime @ Isabelle S.D. Sentis

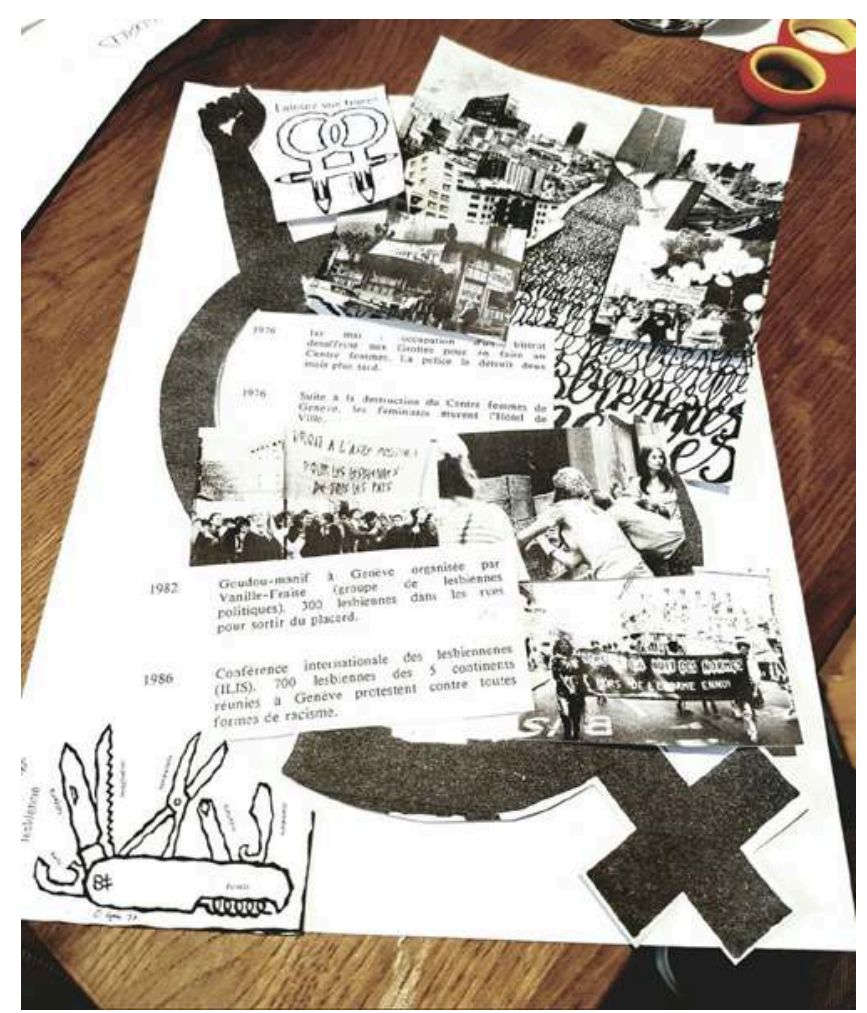

Des références et archives françaises sont entremêlées à des archives genevoises.

Afin de créer une dynamique d'apprentissage collectif, Isabelle S.D Sentis a proposé et animé le format de l'atelier: un dispositif ludique et participatif susceptible de 
favoriser l'implication de chacune, notamment de celles les moins sensibilisées à ces enjeux. Dans le cadre de partage de réflexions et d'émotions de l'atelier, le corps individuel se transforme en corps collectif et politique. Les participantes prennent conscience de leur légitimité à découvrir des archives, à en constituer et à contribuer à leur conservation.

Aux ateliers nous accueillons en moyenne entre vingt et trente participantes. D'autres contribuent en nous envoyant des ressources par mail ou en venant déposer des archives à Lestime. Nous avons choisi de programmer un temps festif au bar associatif de Lestime les vendredis en amont des ateliers se déroulant les samedis. Nous avons baptisé ces vendredis "Les apéros des chattes sauvages", faisant référence aux mythiques «Bals des chattes sauvages» des années 1980 et 1990. Ces rendez-vous ont permis de présenter le projet à des lesbiennes qui ne se voyaient pas participer à des ateliers sur les archives. La musique, constituant le premier des échanges, est une porte d'entrée vers les mémoires. La programmation musicale était préparée en amont par l'une des membres de notre collectif et partait des années 70 jusqu'à aujourd'hui. L'écoute et la danse ont permis des échanges informels, de glaner des informations et des souvenirs. L'un des jeux proposés était par exemple de poser sa bouteille de bière sur la carte à l'endroit de son premier bar lesbien. Quand nous avons mis en place ce dispositif, nous n'avions pas conscience à quel point ces moments seraient centraux dans la mobilisation et la fédération des participantes. Durant les apéros et les ateliers, nous invitions régulièrement les participantes à parcourir les numéros de la revue Clit 007, afin de leur donner envie d'explorer ensuite d'autres types d'archives.

Photo d'ateliers, novembre 2018 (1) @ Isabelle S.D Sentis

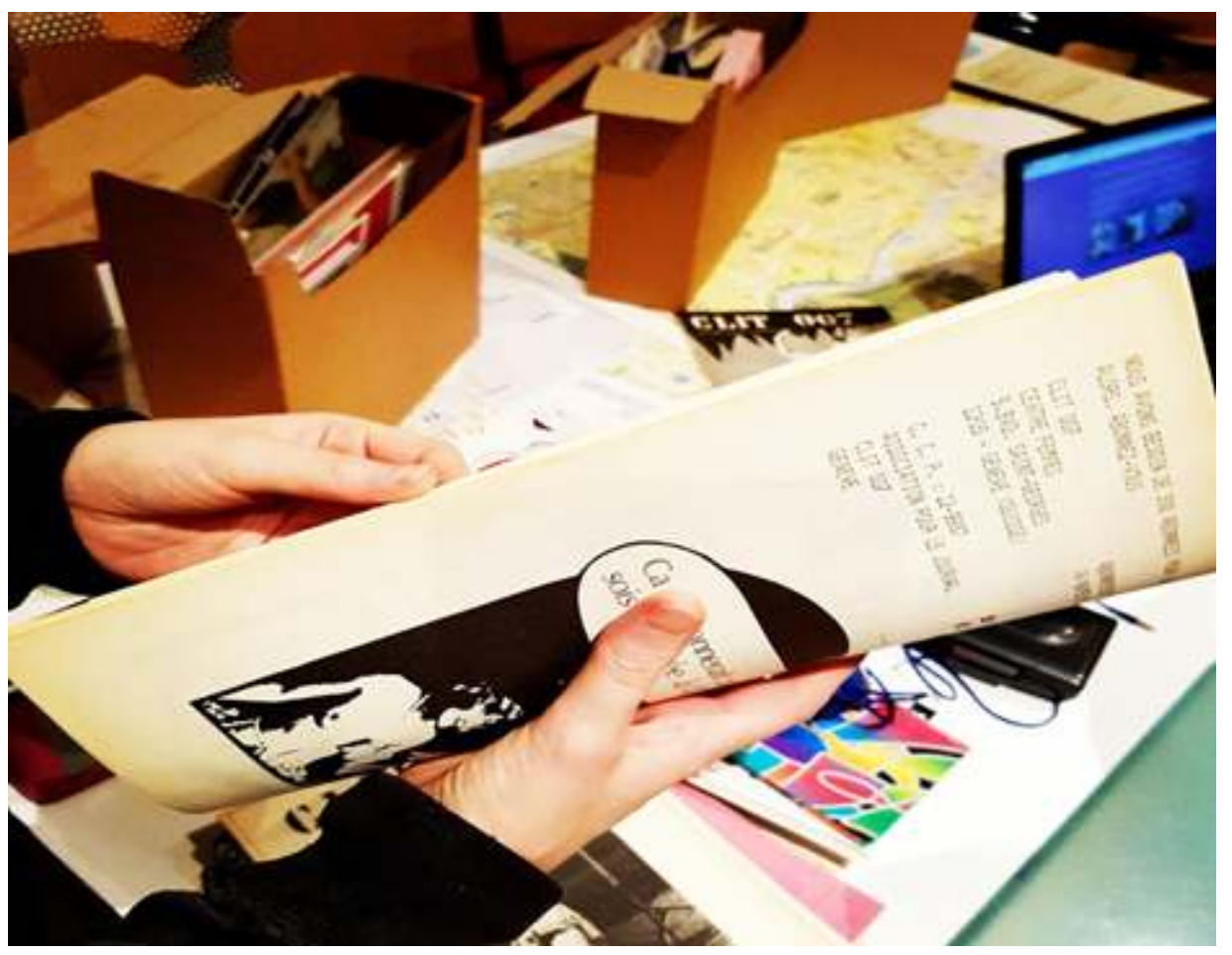


Photo d'ateliers, novembre 2018 (2) @ Isabelle S.D Sentis

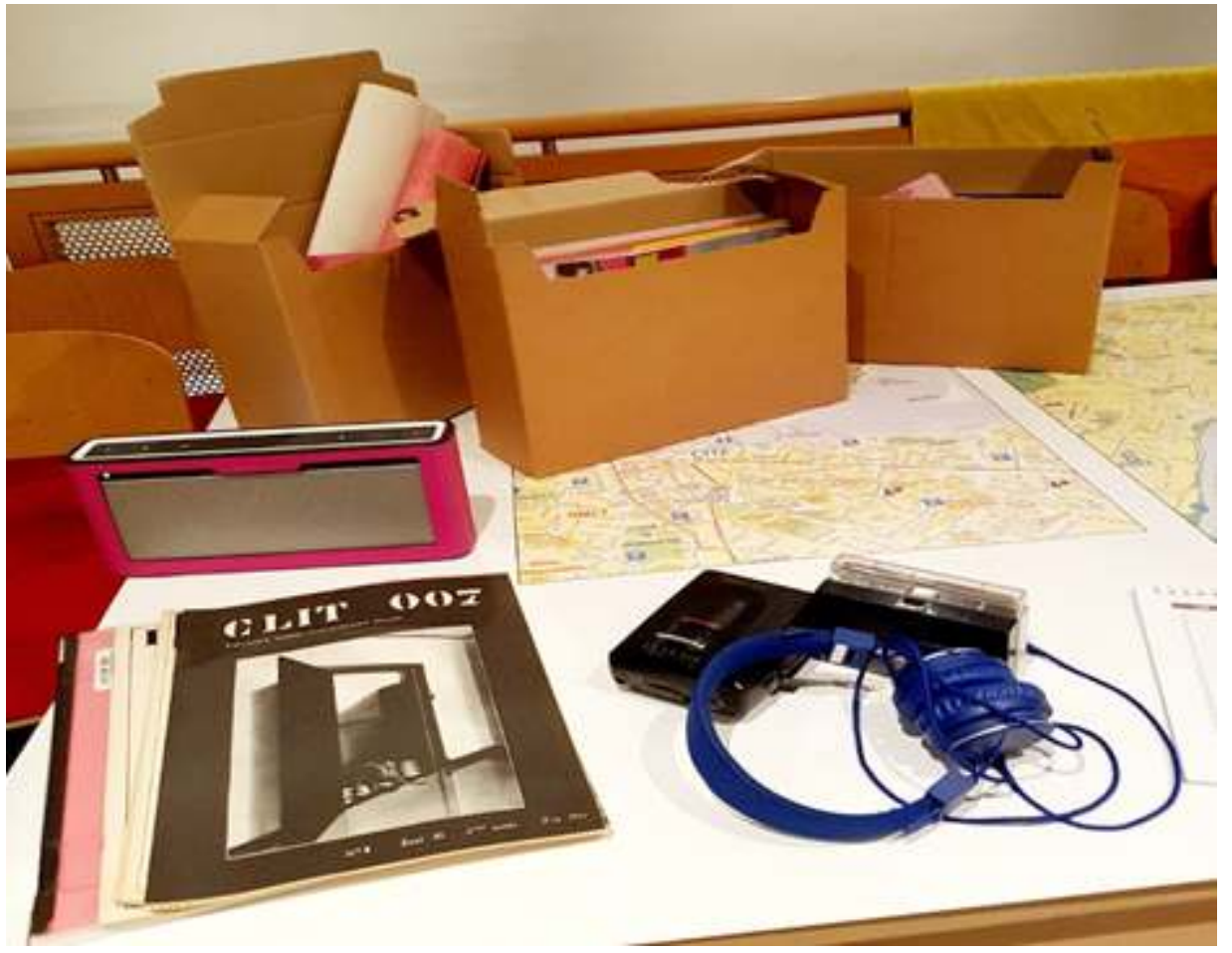

Photo d'ateliers, novembre 2018 (3) @ Isabelle S.D Sentis

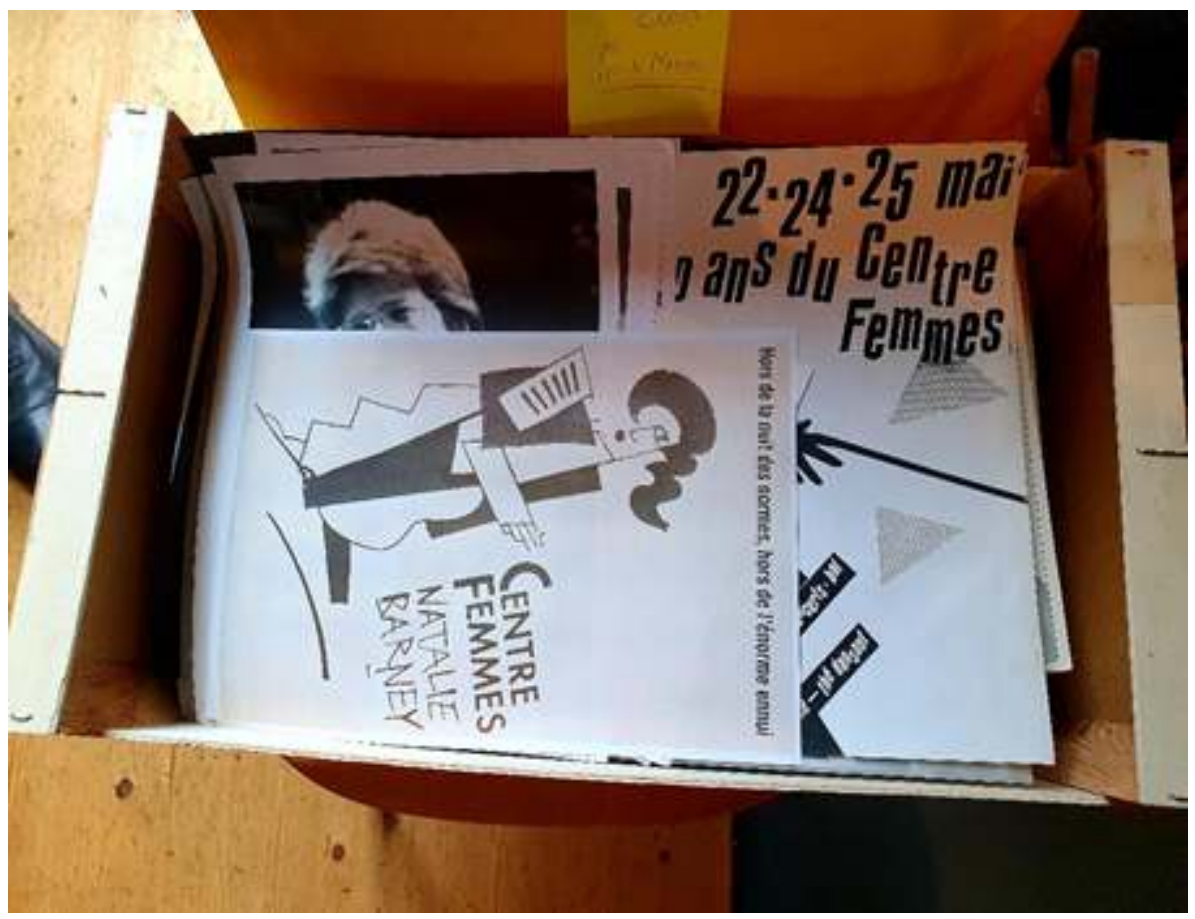


Photo d'ateliers, novembre 2018 (4) @ Isabelle S.D Sentis

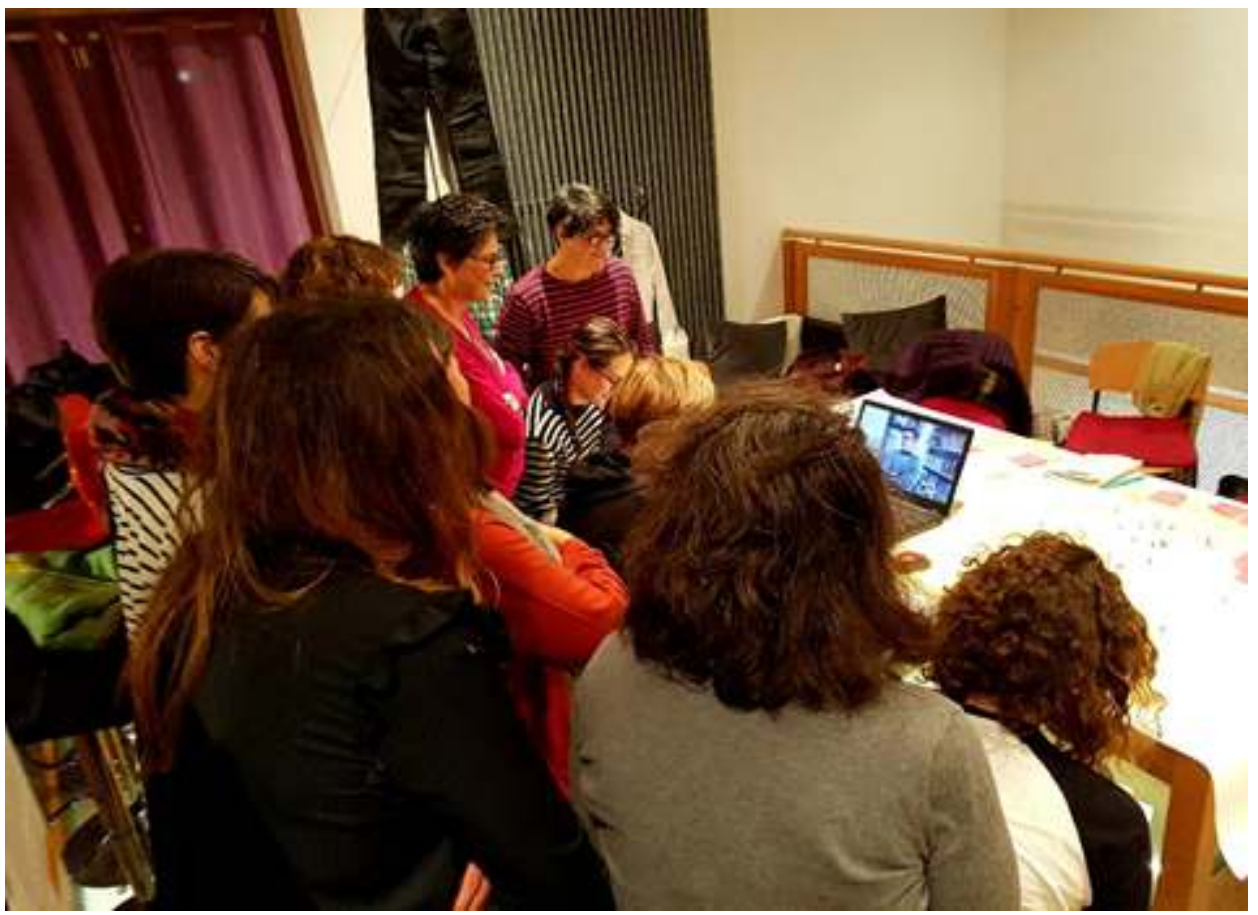

Lors de nos premiers ateliers en novembre 2018 et en février-avril 2019, nous avons travaillé sur l'histoire des cafés, des bars et des boîtes de nuit. Nous avons proposé aux participantes de visionner et commenter collectivement une sélection d'archives de la Radio Télévision Suisse (RTS). Une des membres du collectif, Sophie Meyer, qui est documentaliste à la RTS, a réalisé un mémoire en Études de Genre à l'Université de Genève en 2010 intitulé «Présentation de soi et représentation de l'autre : l'image des femmes homosexuelles dans les émissions de la Télévision Suisse Romande entre 1954 et 2009 ». Son travail a été précieux pour accéder à des sources archivistiques et partager une réflexion critique sur les traitements médiatiques des lesbiennes. Pour retracer l'histoire de La Bretelle, bistrot mythique autrefois fréquenté par les lesbiennes, nous avons réalisé en avril 2019 un entretien collectif à La Bretelle dans une atmosphère chaleureuse. Nos deux interviewées, Kiki et Martine, qui étaient des habituées $d u$ lieu, ont répondu aux questions que les participant-e-x-s avaient préparées en amont par binômes. Le devenir enquêtrice-x a été vécu comme une expérience à la fois formative et ludique. Nous avons remarqué que les interactions qui se sont produites au cours de l'entretien ont réussi à susciter l'intérêt des personnes moins sensibilisées aux enjeux d'archives. Grâce aux souvenirs de Kiki et Martine, nous avons pu dessiner l'ancienne disposition et aménagement du local. Puis, lors d'autres ateliers, Kiki et Martine sont devenues à leurs tours enquêtrice- $\mathrm{x}$ : l'une des attentions du processus coopératif porte sur nos postures et l'invitation à se situer de part et d'autre de l'outil de captation. 
Atelier de glanage à la Bretelle (1), Genève (C) Isabelle S.D Sentis

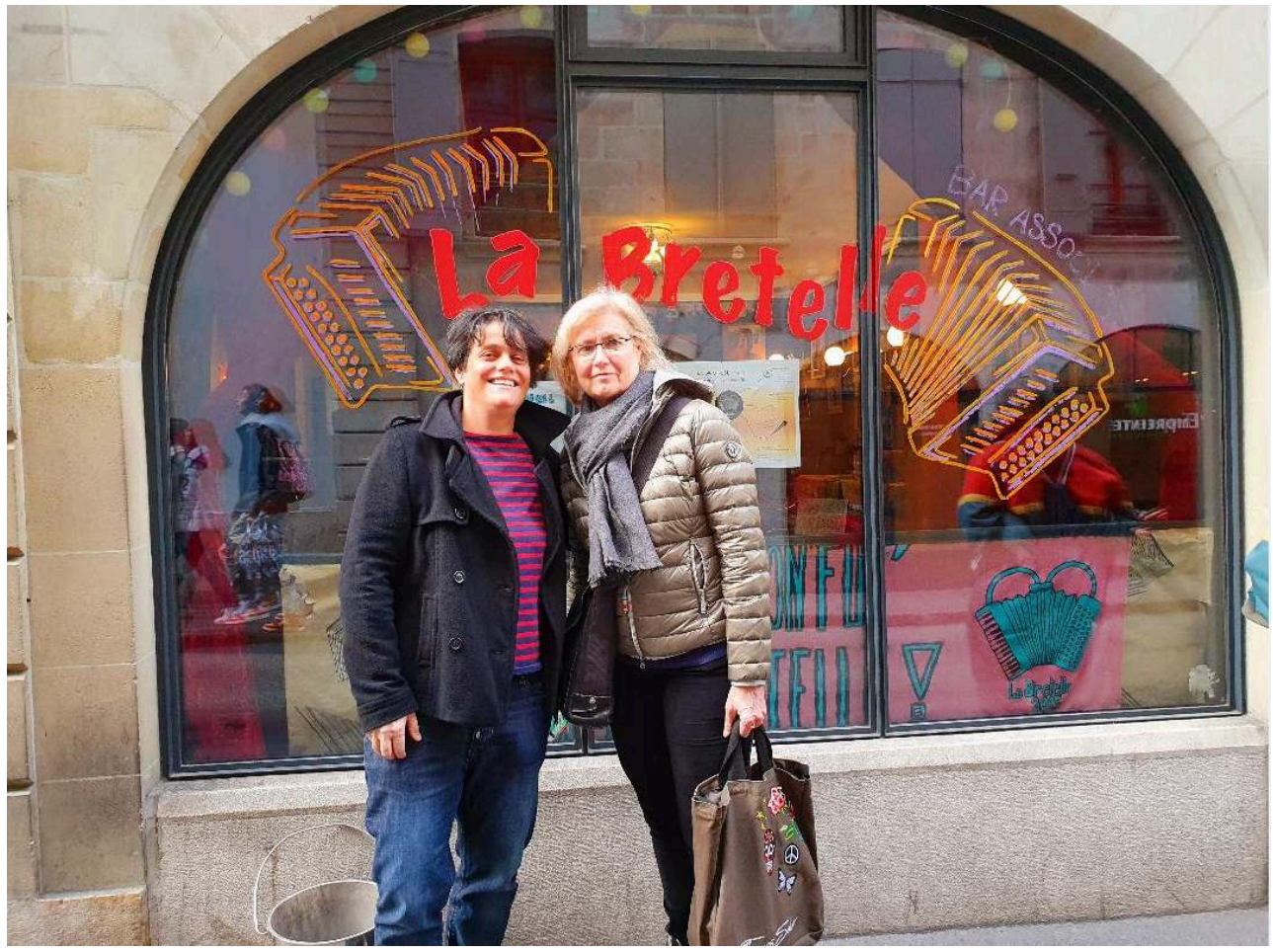

Atelier de glanage à la Bretelle (2), Genève @ Isabelle S.D Sentis

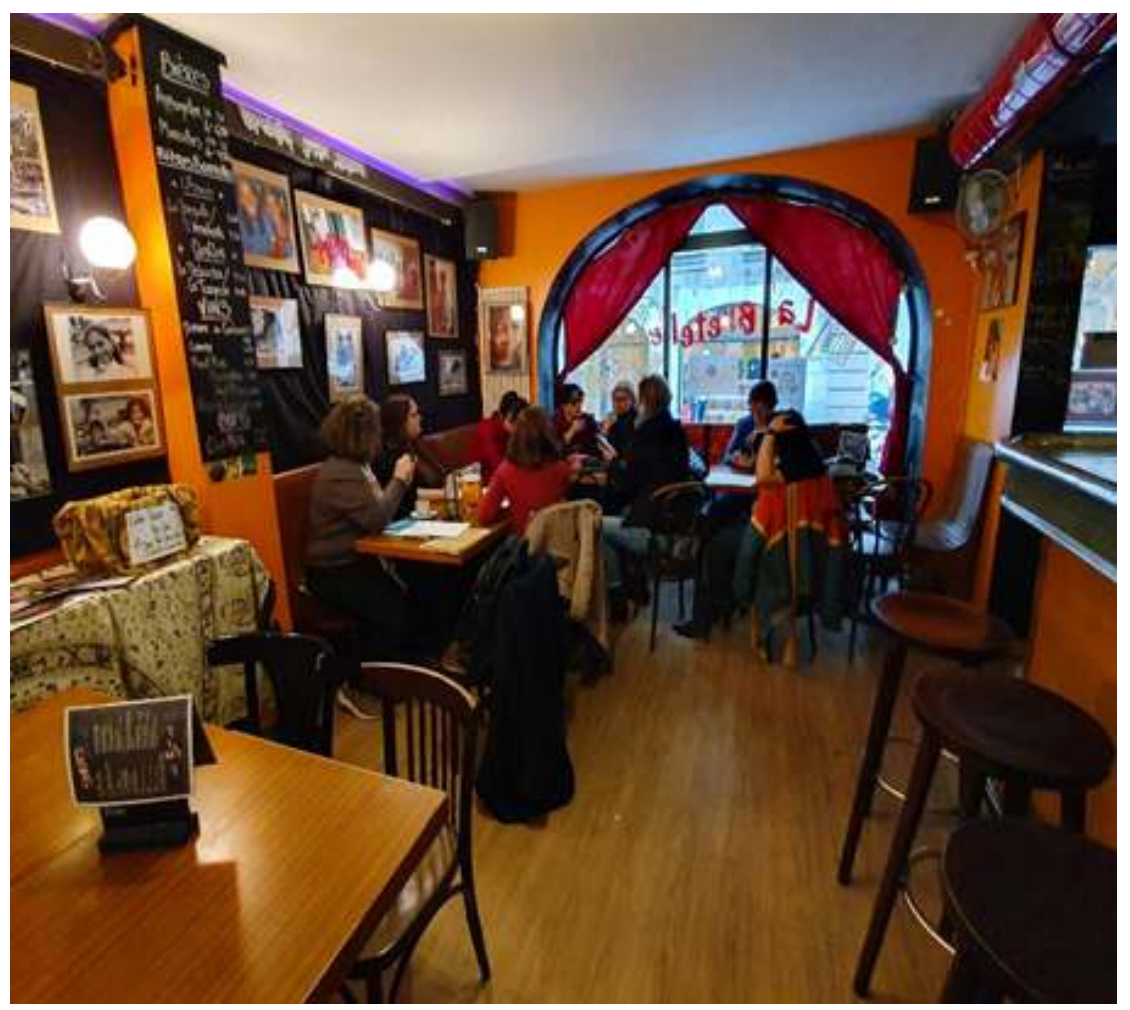


Atelier de glanage à la Bretelle (3), Genève @ Isabelle S.D Sentis

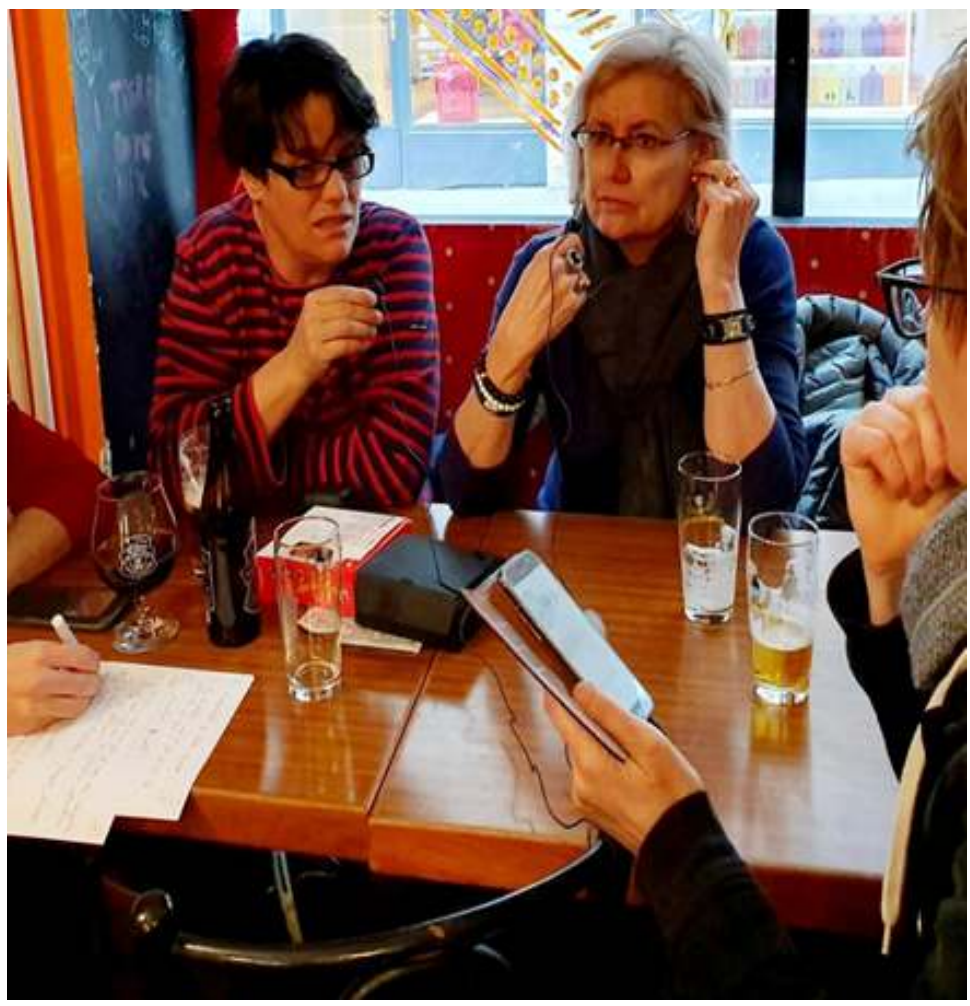

Atelier de glanage à la Bretelle (4), Genève @ Isabelle S.D Sentis

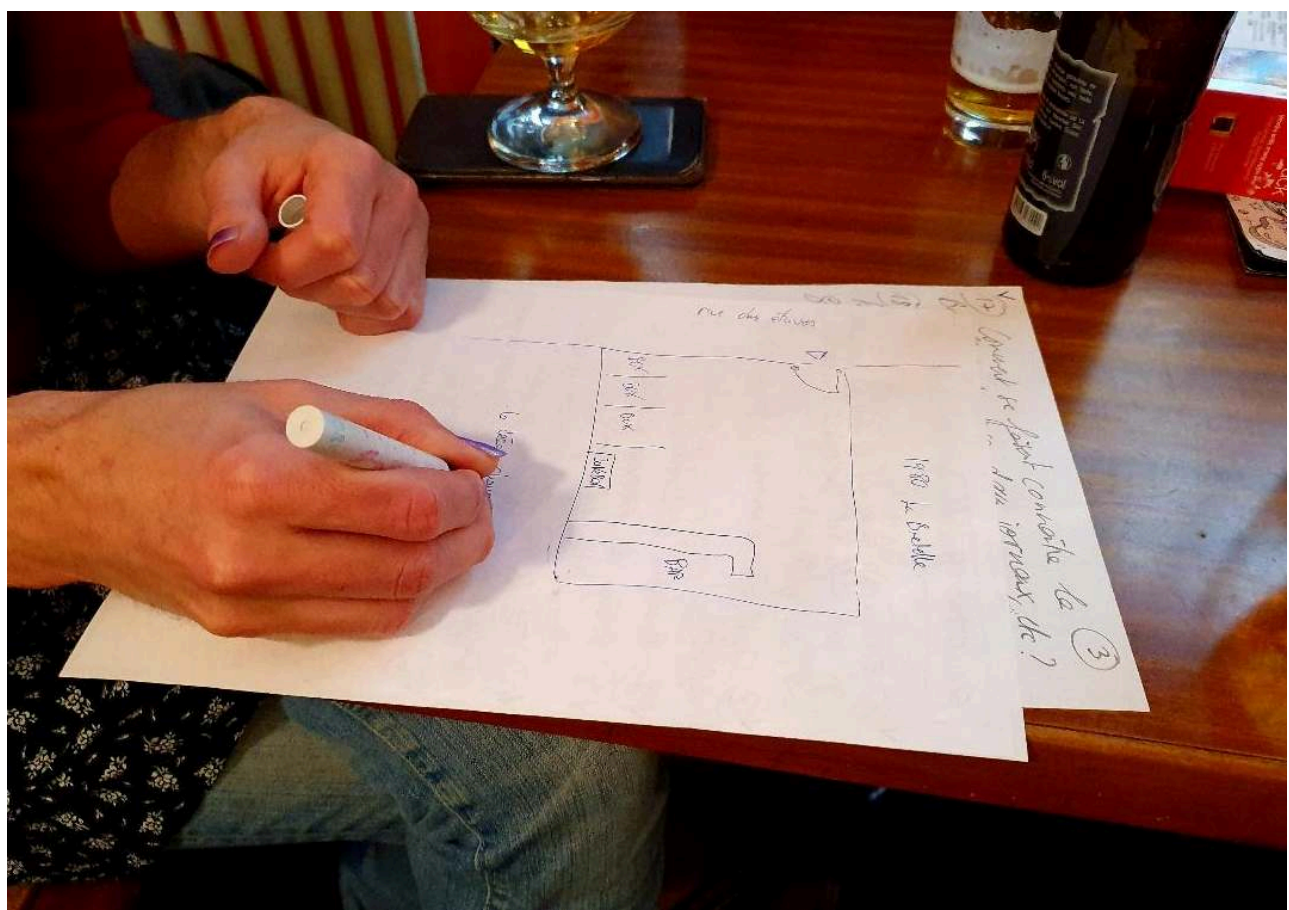

Durant la pandémie, nous avons ensuite organisé deux ateliers thématiques en ligne. En février 2021, l'atelier dédié aux archives des émissions de radio d'hier et d'aujourd'hui a été l'occasion de partager un temps d'écoute collective d'extraits de deux émissions radiophoniques lesbiennes réalisées à Genève dans les années 1980 et 1990 : «Sappho 
Revient» et "Canicule ", et d'échanger sur les pratiques d'écoute d'aujourd'hui (podcasts, émissions, etc.). Cet atelier nous a permis de tisser des liens avec d'autres groupes francophones qui sont en train de collecter et numériser leurs archives d'émissions radio lesbiennes et féministes. En avril 2021, l'atelier dédié aux archives des manifestations a été l'occasion de découvrir les tracts et les photos de quelques évènements marquants de l'histoire lesbienne genevoise, comme la "Goudou-Manif » de 1982 et la marche pour les droits d'asile politique pour les lesbiennes de tous les pays de 1986, faisant le lien avec les revendications actuelles portées notamment lors des marches de nuit du 8 mars et de la Grève féministe. L'objectif était également de sensibiliser les participantes à prendre attention à archiver les tracts, les autocollants et les photos de ces manifestations actuelles.

\section{Collaborations et partenaires}

Le collectif Notre histoire compte a mis en place plusieurs partenariats et collaborations. Premièrement, nous avons coopéré avec la Ville de Genève lors de sa campagne 2019 sur le thème des "Mémoires LGBTIQ+", pour visibiliser la riche histoire locale des mouvements. Lorraine Astier, Carolina Topini et Mathilde Matras, ont été mandatées pour réaliser la première enquête exploratoire sur les archives des luttes LGBTIQ à Genève (années 1970-2000) ${ }^{5}$, dont le rapport est disponible en ligne. Une conférence intitulée "Quand l'histoire LGBTIQ+ genevoise s'affiche» a été donnée par Sophie Meyer dans le cadre des "Jeudi midi de l'affiche » de la bibliothèque de Genève. En puisant dans la collection d'affiches de la bibliothèque de Genève ainsi que dans les archives de différentes associations locales, l'objectif était de montrer que l'histoire des luttes LGBTIQ+ s'est également écrite sur les murs. L'année 2019 célébrait également le retour de la Pride romande à Genève ainsi que les 50 ans des Emeutes de Stonewall. Dans ce contexte de commémoration, nous avons participé à la journée de réflexion du 17 mai contre les lgbt-phobies "L'histoire fait son coming-out », organisé à l'Université de Genève. Un atelier de "Speed dating avec des archives LGBTQI* " a été proposé et animé par Isabelle S.D. Sentis qui invitait à la découverte partagée d'archives. Nos archives nous ont également amenées à reprendre la rue : dans le cadre de la Geneva Pride 2019, nous avons proposé la "Balade au cœur de notre histoire", une visite guidée des lieux militants et festifs des années 1970 à 2000 relatifs principalement au mouvement et à la culture lesbienne.

Un partenariat a été ensuite établi avec la Radio Télévision Suisse (RTS), afin de pouvoir faire découvrir une sélection de leurs archives audiovisuelles. Une autre collaboration importante autour des archives des radios féministes et lesbiennes a été établie avec les Archives contestataires de Carouge, où se trouvent plusieurs fonds concernant l'histoire du MLF et du mouvement lesbien genevois des années 1970. Un partenariat avec la Bibliothèque Municipale de Genève s'est concrétisé dans l'organisation conjointe d'un atelier de cartographie lesbienne et bisexuelle animé par les membres de Queer Code, ayant eu lieu le 22 mai dans le cadre de la campagne 2021 contre l'homophobie, la biphobie et la transphobie de la Ville de Genève. Tout au long du projet, nous avons collaboré avec l'association Les Sans Pages pour réaliser des articles wikipédia sur les biographies des femmes et de groupes qui ont marqué l'histoire LBT+ locale. 
Le 11 novembre 2020, Lestime a organisé une table ronde en ligne intitulée «Les archiveuses », pour réfléchir sur la transmission des archives féministes et lesbiennes et célébrer le travail de traitement de ses archives réalisé en 2020 en collaboration avec deux archivistes professionnelles. Le 19 octobre 2021, une table ronde sur « Histoires et mémoires LGBTIQ+ : imaginer l'archive du futur » a été proposée par Lestime, la Ville de Genève, l'Institut des Études genre et la Maison de l'Histoire de l'Université de Genève, afin de (re)penser collectivement les pratiques de conservation, de valorisation et de transmission du patrimoine matériel et immatériel des (sub)cultures LGBTIQ+.

Nous tissons petit à petit des liens avec d'autres collectifs et structures LGBTQI+ qui mènent aux États-Unis, au Canada, en Grande-Bretagne et en France des projets proches du nôtre. D'autres partenariats sont envisagés par notre collectif à une échelle locale et internationale.

\section{Une balade virtuelle à la découverte de nos archives}

\section{Pour commencer : quelques repères sur l'histoire des mouvements lesbiens à Genève}

Avant de commencer notre propre recherche, nous nous sommes documentées sur l'histoire des lesbiennes genevoises auprès du centre de documentation de Lestime. C'est ainsi que nous avons découvert qu'Helene Joly avait réalisé en 1998 un mémoire de master en Études genre à l'Université de Genève intitulé «De Sappho s'en fout à Vanille fraise (1972-1986): Histoire du mouvement lesbien genevois». Il s'agit du premier travail (et le seul à ce jour) sur la trajectoire du lesbianisme politique à Genève'.

D'après Joly, le rôle du Mouvement de Libération des femmes (MLF) a été crucial dans le processus d'affirmation lesbienne. Il permet la constitution d'un espace de sociabilité politique « entre femmes ", où chacune peut s'exprimer à partir de son vécu. Parmi les pionnières genevoises du MLF il y a un nombre important de lesbiennes. C'est au MLF que de nombreuses femmes se découvrent lesbiennes; tandis que d'autres, qui se considéraient déjà comme telles, y trouvent "un lieu de parole privilégié ». Les lesbiennes furent les premières à interroger ce récit idyllique, parfois reconduit par l'historiographie. En effet, selon un schéma très courant dans d'autres contextes, un mouvement lesbien autonome émerge à Genève quelques années après la constitution du MLF, sur la base d'une série de conflits entre militantes lesbiennes et hétérosexuelles. Les lesbiennes remettent notamment en question les idéaux de "sororité » et la prétendue homogénéité d'oppression entre les femmes, reprochant aux hétérosexuelles leur manque de soutien et dénonçant le contournement du lesbianisme de la part des soi-disant "hétéroféministes ». Les féministes préfèrent garder dans leurs tracts l'appellation plus respectable de « femmes » : « lesbienne » est un mot très lourd à assumer (Joly, 1998: 7). L'héritage du MLF reste néanmoins perceptible dans le nouveau mouvement lesbien: dans la permanence des mêmes militantes qui s'y sont formées, mais aussi dans les formes d'organisation qui en préservent le caractère spontanéiste, le même refus de structures rigides et de leaders officiels (Joly, $1998: 8$ ).

Selon la chronologie proposée par Joly, la publication en 1972 d'un pamphlet intitulé " Hétéros on est navrées de vous gêner!» peut être considérée l'acte fondateur d'un 
mouvement genevois de lesbiennes féministes. Le texte aborde de manière polémique la place réservée aux lesbiennes au sein du MLF et s'inspire de la radicalité du groupe parisien des Gouines Rouges, que les militantes genevoises avaient rencontré quelques mois avant lors des Journées de dénonciation des crimes contre les femmes à la Mutualité de Paris. Les contacts avec les militantes à l'étranger sont multiples, même si ce sera seulement à travers le groupe de lesbiennes politiques de Vanille Fraise, né en 1979 de la dissolution du collectif L'Insoumise, que Genève s'affiche dans le mouvement lesbien international. Le groupe se nourrit des idées et des pratiques des mouvements internationaux, participant à l'émergence d'un réseau transnational d'échanges. Le groupe est également très actif au niveau local. Il concentre ses efforts principalement dans la publication d'un journal/fanzine trimestriel, Clit 007. Concentré Lesbien Irrésistiblement Toxique (1980-1986), et dans la réalisation d'émissions radiophoniques pirates. Les militantes de Vanille Fraise s'affirment sur la scène publique organisant des manifestations autonomes, dont la "Goudou-Manif » du 22 mai 1982 à Genève, et distribuant des tracts où la provocation et l'humour sont à l'ordre du jour. Parallèlement elles travaillent à développer une sociabilité locale organisant régulièrement des fêtes (les fameux 'Bals des chattes sauvages') et développant des liens nationaux et internationaux avec les autres groupes lesbiens qui se développent en Europe et aux États-Unis entre les années 1970 et les années 1980. L'alliance avec le mouvement lesbien international se concrétise en 1984, quand les lesbiennes genevoises prennent en charge le secrétariat de l'International Lesbian Information Service (ILIS) jusqu'à 1986.

La fin de la revue Clit 007 en 1986, qui était entretemps devenue un point de référence pour la communauté lesbienne francophone, marque selon Joly " la fin d'une époque » et le commencement d'une " phase de transition » $(1998: 13)$. Le mouvement change de peau et rentre dans une phase d'institutionnalisation qui le transforme en profondeur. Après avoir atteint son apogée dans la première partie des années 1980, le mouvement commence à perdre sa "capacité mobilisatrice» (1998: 18). D’après les récits dominants sur les vagues et les périodisations féministes, les années 1980 sont censées marquer une sorte de repli, un moment d'essoufflement de l'action féministe après le foisonnement des années 1970 : le fameux « creux de la vague ». Notre recherche nous a permis d'aller au-delà d'un certain nombre d'idées reçues sur cette période et de redécouvrir toute sa richesse politique, notamment pour ce qui concerne l'émergence d'un militantisme spécifiquement lesbien à visée internationale qui n'obéit pas à la même chronologie qui fait des années 1980-1990 un moment de reflux.

\section{"Les femmes construisent, la ville démolit »... Le MLF en colère}

Le fait de pouvoir disposer d'un espace "à soi » est ressenti depuis le début par les militantes lesbiennes et hétérosexuelles du MLF comme une priorité. En 1975, elles demandent en plusieurs occasions à la maire de Genève de mettre à leur disposition un local pour les réunions, argumentant sur le rôle social que le MLF remplit vis-à-vis des femmes en détresse. Lassées d'attendre la réponse des pouvoirs publics, le $1^{\circ}$ mai 1976 une centaine de militantes occupent un vieux bistrot désaffecté dans le quartier populaire des Grottes. Malgré les demandes de la Ville, les femmes refusent de le quitter. La Ville décide alors de porter plainte et les inculpe de violation de domicile. Le 10 août à l'aube, les militantes sont expulsées par la police et le local des Grottes est en partie démoli à la pelle mécanique. Deux jours après, le 12 août elles décident de mener 
une action spectaculaire en guise de représailles : munies de briques, de ciment et de truelles, elles partent en cortège jusqu'en vieille ville de Genève. Une fois arrivées devant le siège du Conseil administratif, elles en murent l'entrée. Des manifestes affichant les slogans « Nous sommes toutes des occupantes ", « Le MLF construit, la ville démolit ", "Un centre femmes pour nous organiser ", sont mis en circulation pendant la manifestation, qui compte environ 300 personnes. En 1980, l'émission Ouvertures consacre une édition spéciale à l'histoire du MLF sous le titre " Les mouvements féministes, le temps des sorcières ", revenant notamment sur cet épisode.

Photogramme du reportage "Nous voulons un centre femmes », 4 mai 1976, archives en ligne de la RTS.

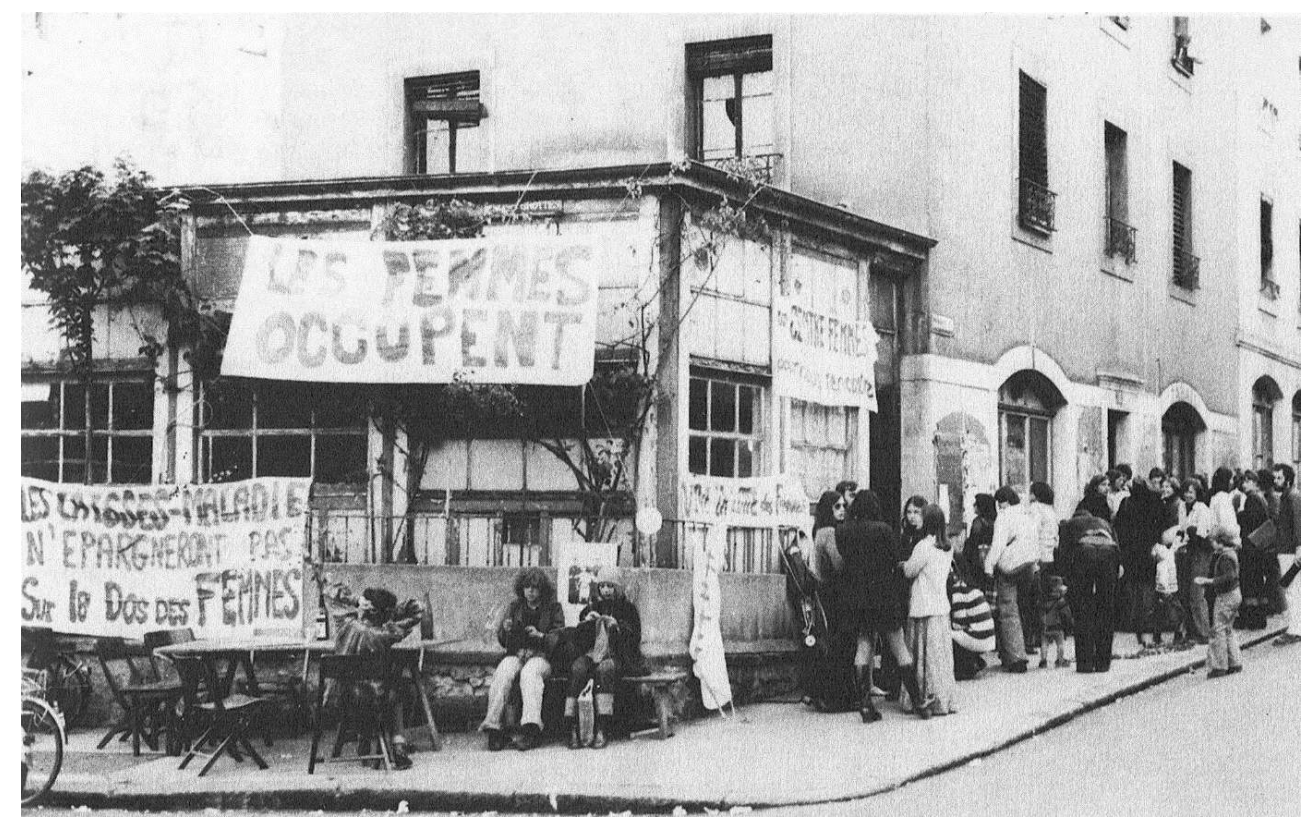

Photogramme du reportage « Genève, le MLF en colère » (1), 13 août 1976, archives en ligne de la RTS

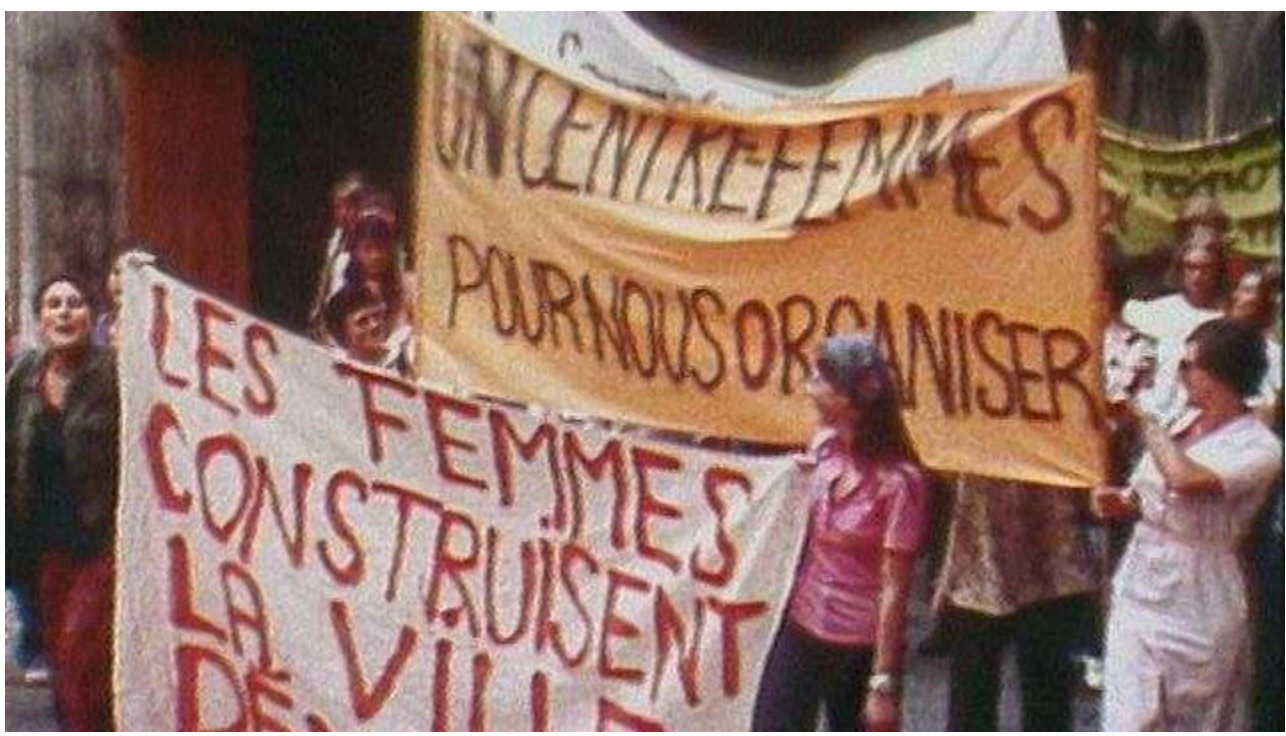



RTS

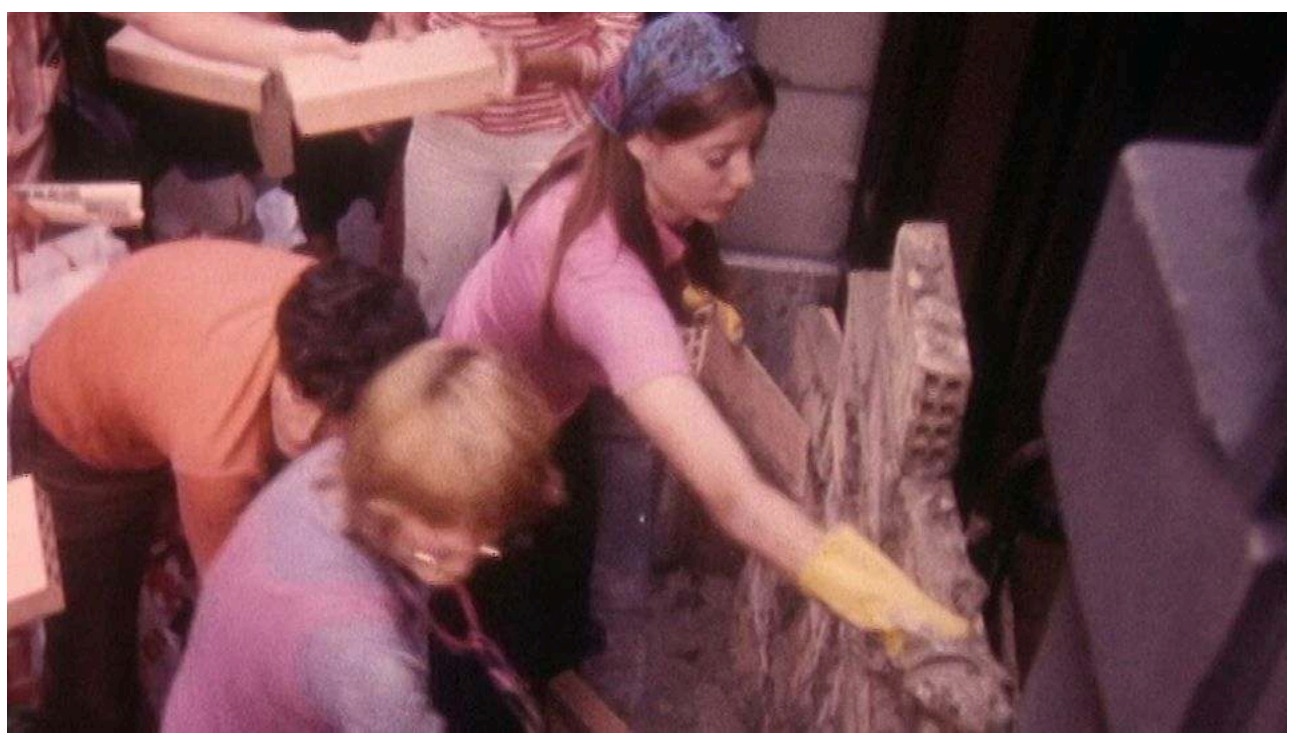

Après la démolition du local des Grottes, les féministes attendent février 1977 pour se voir enfin attribuer un local au rez-de-chaussée du 5 boulevard St-Georges. Si pendant quelques temps plusieurs associations se partagent l'espace, les lesbiennes vont rapidement être les seules occupantes. L'attribution du centre ne sera plus contestée jusqu'à 1989, quand il fermera ses portes.

\section{La fête est politique : les «Bals des chattes sauvages »}

Voix off : Patti Smith, Horses, full album, 1975

Voix off : Eurythmics, Sweet Dreams, full album, 1983

Lieu d'accueil et d'entraide pour les lesbiennes de Genève, le Centre femmes du boulevard St-Georges est également le cadre d'un rassemblement festif mythique dans le milieu lesbien : le « Bal des chattes sauvages ». Tous les premiers samedis du mois, les femmes ont la possibilité de danser entre elles dans un lieu protégé à l'abri des regards voyeurs, oubliant le temps d'une soirée l'hostilité du monde extérieur. Le développement d'un pôle militant plus festif répond à la volonté de Vanille Fraise d'atteindre les soi-disant « lesbiennes des bars » et pas seulement celles déjà politisées. Il s'agit donc d'une précise stratégie de conscientisation et d'ouverture, participant à atténuer les frictions entre les "homos fêtards » et les "femmes féministes ". L'importance de toucher l'ensemble des lesbiennes, était d'ailleurs partagée par d'autres groupes féministes et lesbiens de l'époque, dont les Gouines Rouges, qui comme nous raconte Marie-Jo Bonnet, avaient l'habitude de distribuer leurs tracts à l'entrée de boites de nuit parisiennes (1998: 94).

Le premier bal des chattes sauvages est organisé au Centre femmes en février 1982. D'après une des militantes interviewées par Helene Joly, pendant les premiers bals, des interventions politiques étaient régulièrement effectuées par Vanille Fraise (1998: 76). La fête est perçue comme un moment fédérateur susceptible de contribuer indirectement à des mobilisations proprement politiques. L'organisation de fêtes, bals 
et concerts permet en effet à une pluralité de femmes d'horizons divers de se connaitre, contribuant à établir et renforcer des réseaux lesbiens tant à un niveau local qu'international. À l'occasion du Tortilla Sax Festival, organisé par Vanille Fraise les 11-12 mai 1984, environ 250 femmes venues de toute l'Europe se réunissent au centre de loisirs de Carouge autour d'un riche programme musical, qui compte entre autres le groupe Cobrha de Paris et les Miami Beach Girls d'Amsterdam.

Pendant nos ateliers, nous avons réalisé deux entretiens audio-visuels avec Lorena Parini (autrement connue comme DJ Lap), qui avec sa musique a animé de nombreux bals des chattes sauvages au Centre Femmes de Boulevard Saint-Georges. Les membres du collectif ont souhaité créer deux dispositifs d'interview : le premier dans le vieux local du Centre Femmes, devenu aujourd'hui une épicerie, afin de créer une archive in situ; le deuxième chez Lorena dans une ambiance plus intime. Dans son témoignage, disponible via la cartographie Notre histoire compte, elle nous présente deux chanteuses et artistes phares dans sa playlist : Patty Smith et Annie Lennox.

Nous vous proposons de parcourir les numéros de la revue Clit 007 pour retrouver des affiches et articles de ces soirées.

Clit 007, n. 5 décembre 1982

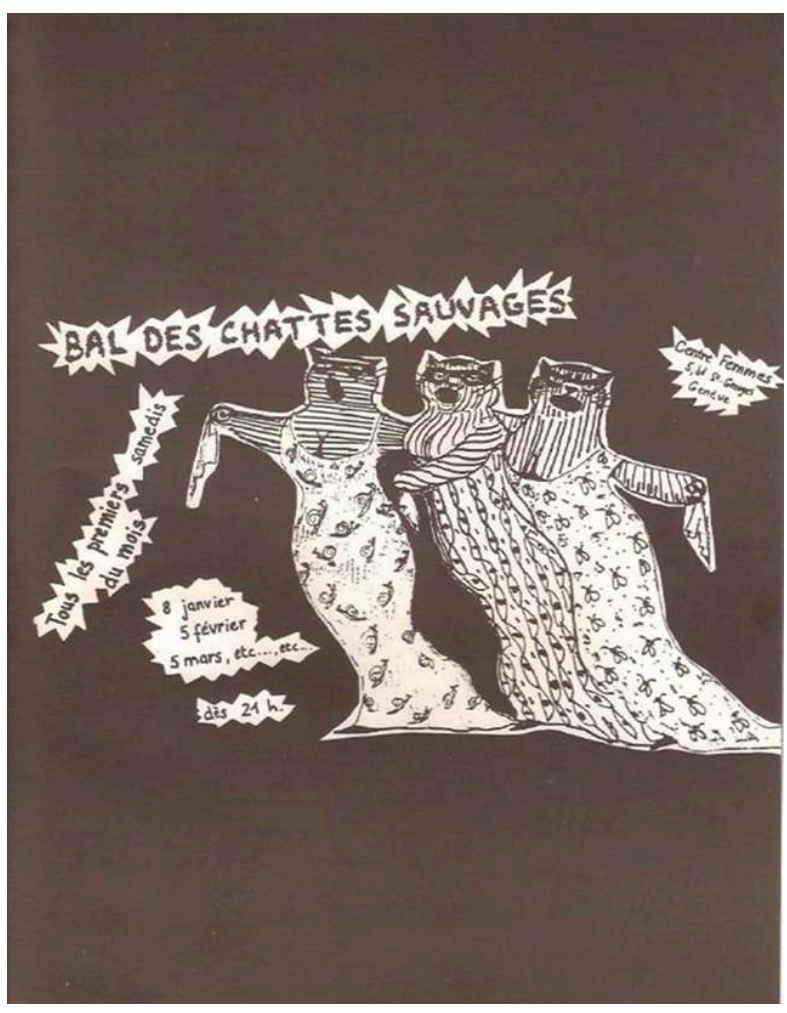


Clit 007, n. 6 mars 1983

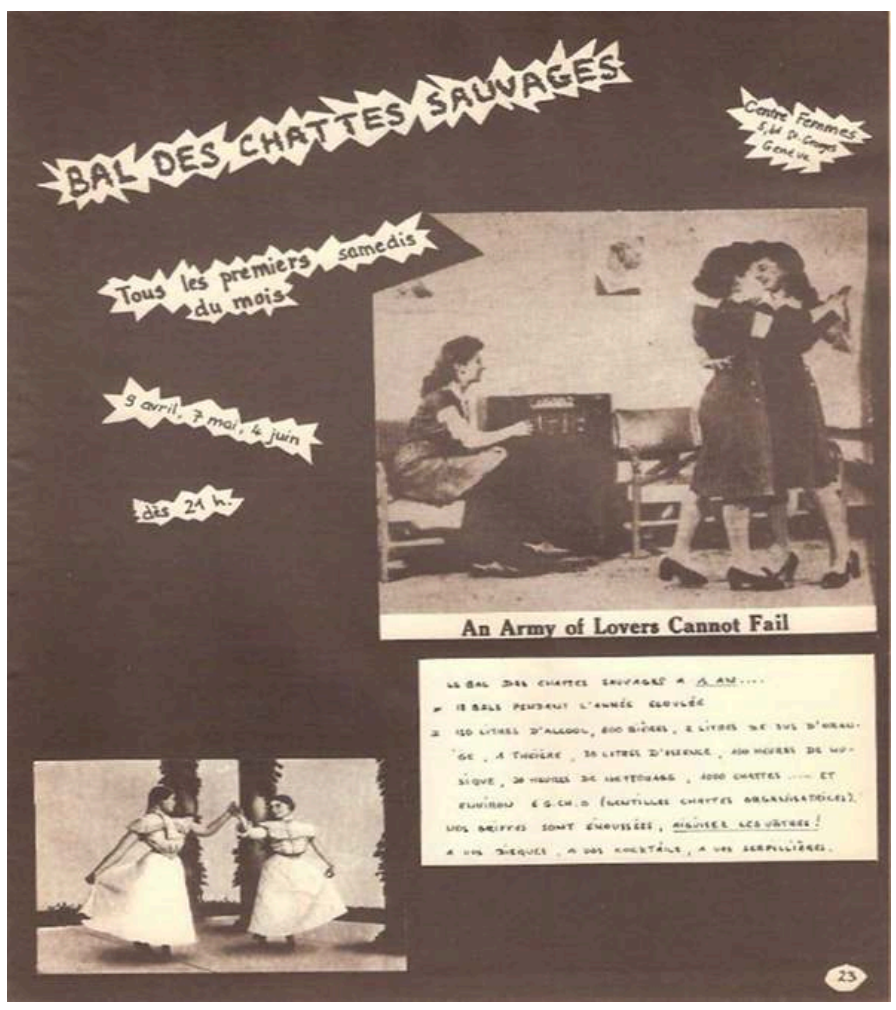

Clit 007, n. 10 mars 1984

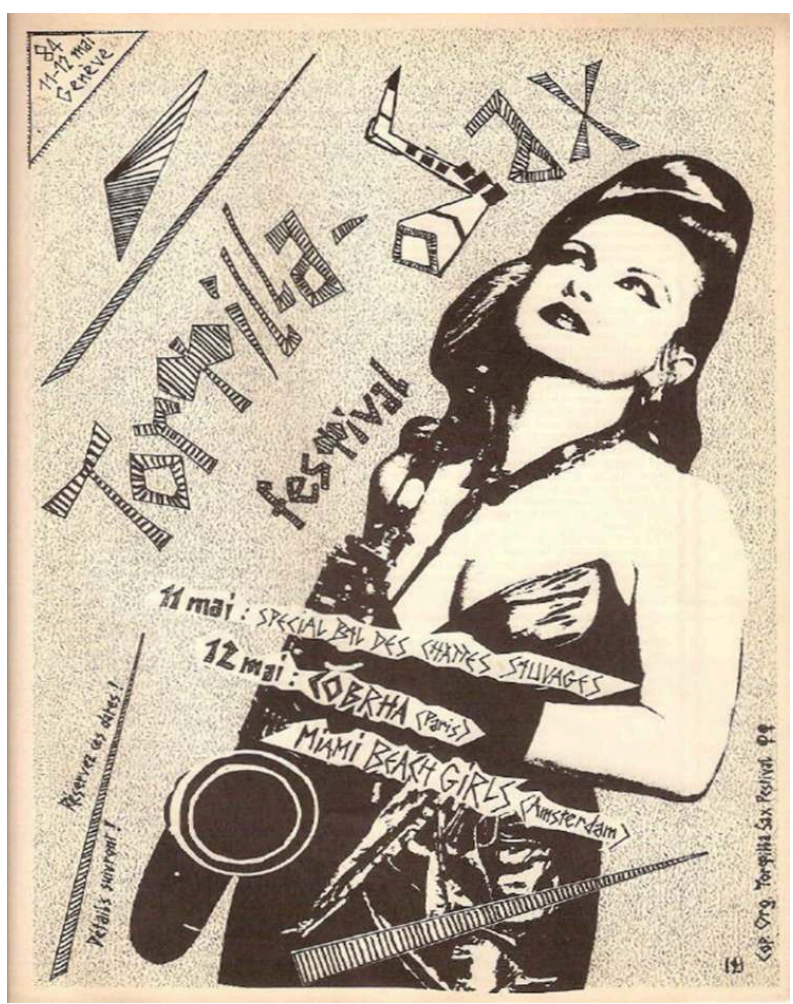


Clit 007, n. 12 septembre 1984 (1)
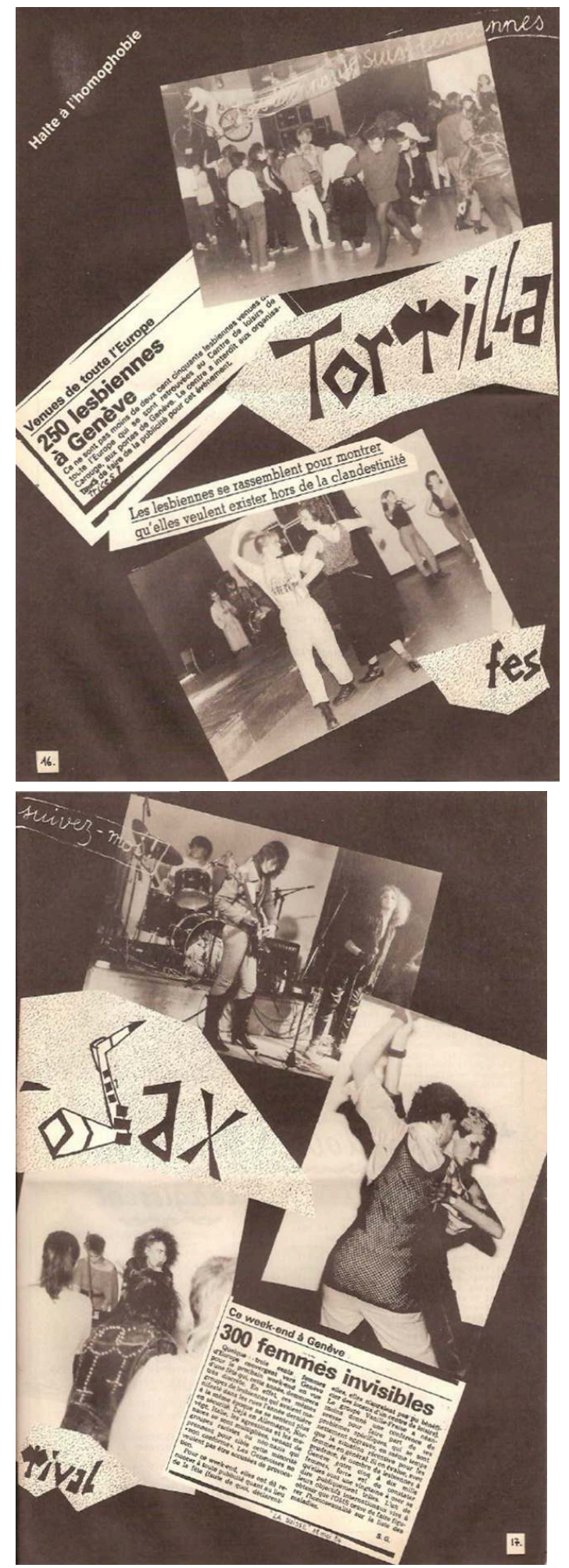


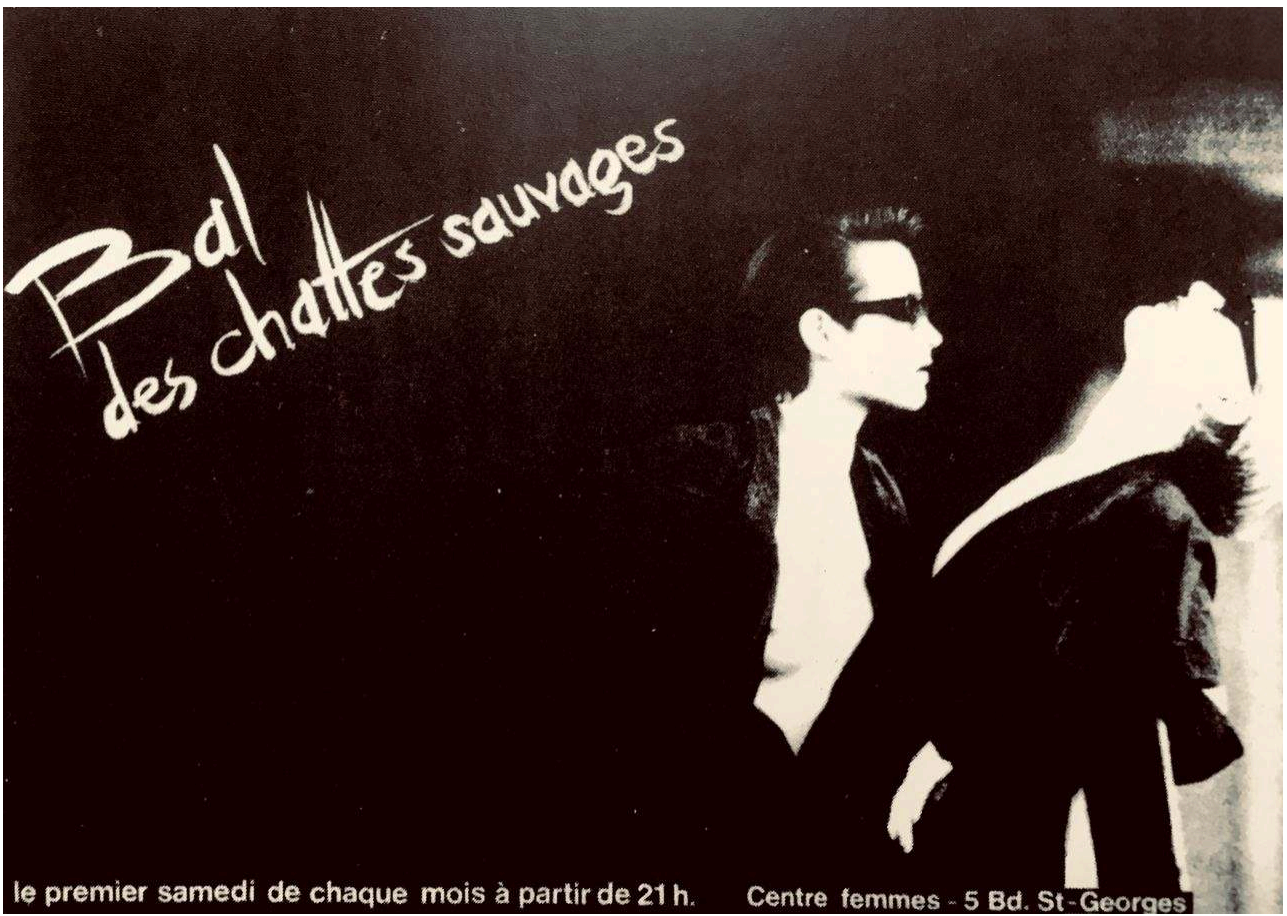

\section{La « Goudou-Manif »: la première manifestation en non-mixité}

Le samedi 22 mai 1982 « un fort courant chaud de lesbiennes perturbe l'ouest de la Suisse ", notamment la ville de Genève... il s'agit de la "Goudou-Manif », la première manifestation publique non mixte des lesbiennes de terre helvétique. Une centaine de lesbiennes défile dans les rues de Genève au son des tam-tams et autres instruments de musique, selon des répertoires typiques des mobilisations collectives (musique, dance). Le cortège part de la place des Alpes. La banderole de tête affiche un slogan resté célèbre : «Hors de la nuit des normes, hors de l'énorme ennui ». D’autres banderoles proposent des slogans volontairement ironiques et provocateurs : «Si on peut envoyer un homme sur la lune, pourquoi ne pas les y envoyer tous? ", "Les femmes dans nos bras, pas dans leur cuisine ", "Lesbiennes ne portez plus le ciel sur vos épaules, demandez la lune! ", "Vive la libre circulation, à bas les ghettos ". Certaines manifestantes défilent à visage découvert, tandis que d'autres portent des masques ou des lunettes noires, comme on a pu le voir dans un reportage réalisé par la RTS projeté lors de nos ateliers. Au début des années 1980, le placard est encore une réalité pour de nombreuses lesbiennes.

La manifestation est suivie d'un bal au Centre de loisirs du quartier de la Jonction. La journée du dimanche est en revanche consacrée à des ateliers de discussion politique, création culturelle et Wendo, une technique d'autodéfense inspirée des arts martiaux et destinée spécifiquement aux femmes (Joly, 1998 : 85). La manifestation est organisée par différents collectifs lesbiens dont le FLOH (Frauen lesbich oder Homosexuellen) de Zürich, le groupe Vanille-Fraise de Genève, les Brigades Roses Neuchâtel, ainsi que par d'autres militantes de Bâle, Fribourg et Berne. Elle se veut une action de visibilité destinée à faire prendre conscience aux lesbiennes de leur puissance en tant que groupe. Son ampleur est nationale et même internationale: des femmes provenant 
d'Allemagne, de France et d'Italie défilent avec les Suissesses en signe de solidarité. Nous avons diffusé en mars 2021 un appel à témoignage et à archives pour reconstruire la mémoire de cette manifestation. Les entretiens et la collecte d'archives sont toujours en cours.

Un autocollant distribué lors de la Goudou-Manif, retrouvé dans un journal intime confié après le décès de son autrice à une des membres de notre collectif.

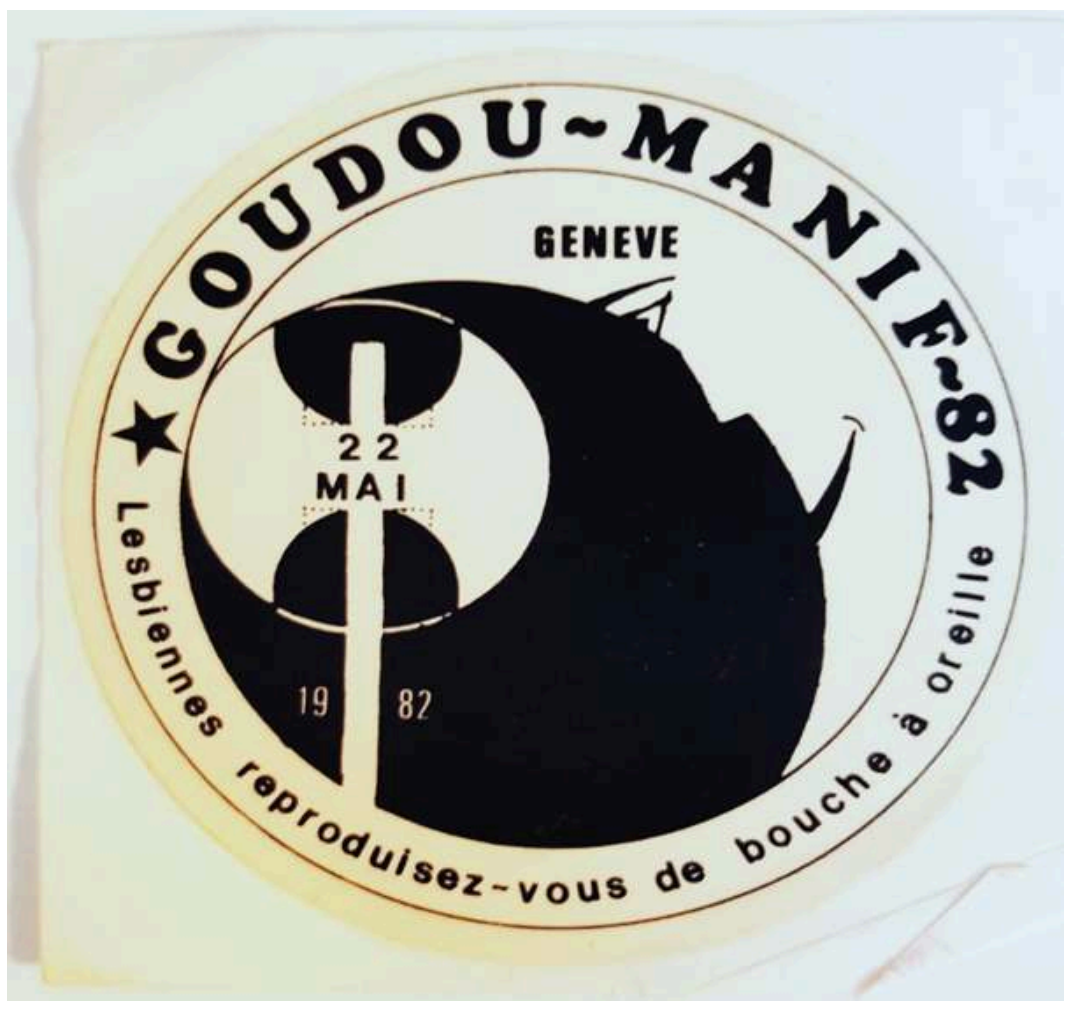


Photographie de la bannière principale de la Goudou-Manif « Hors de la nuit des normes, hors de l'énorme ennui ». Fonds privé de Marie de Montmollin @ RTS

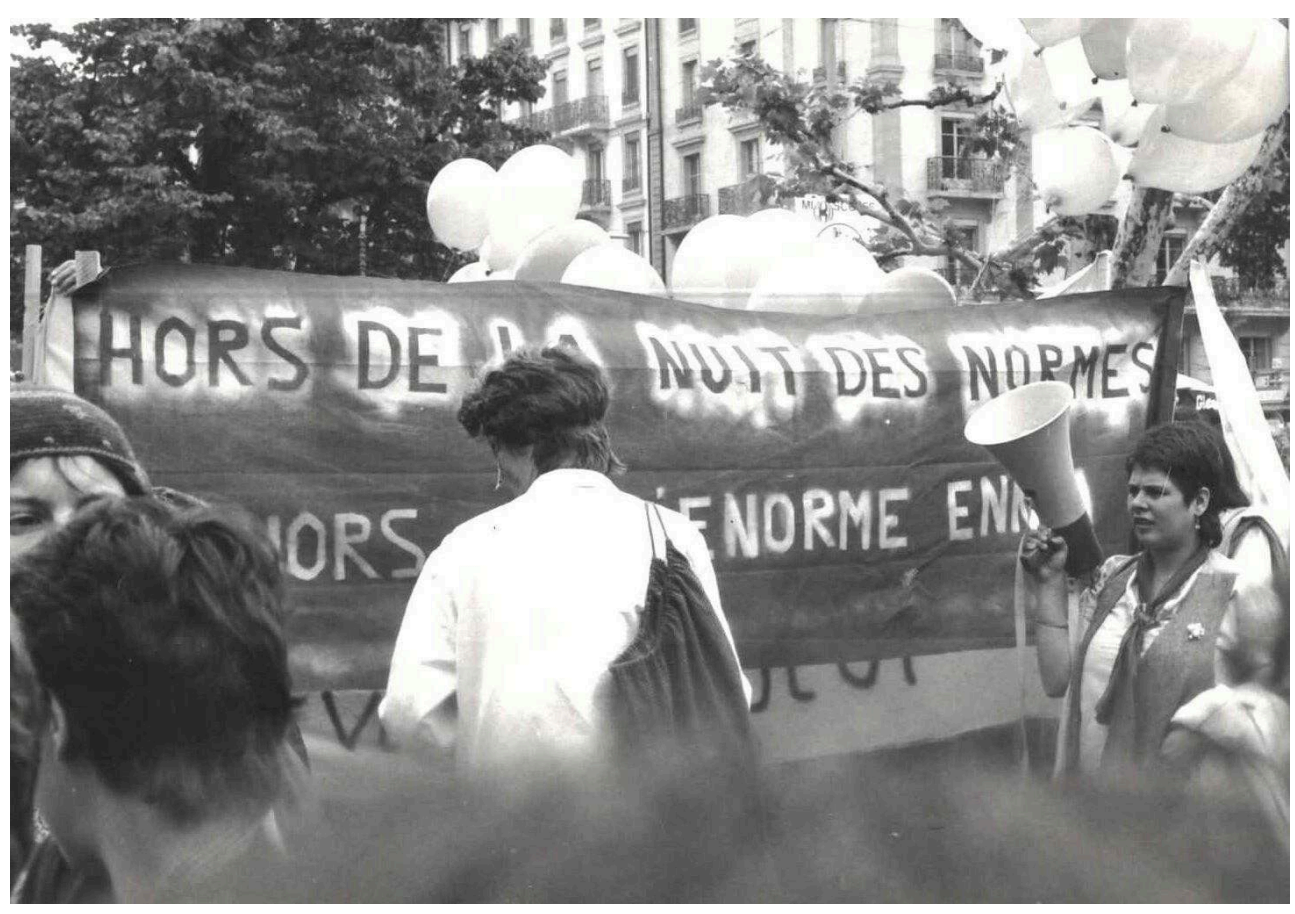

Affiche de la Goudou Manif numérisée et conservée aux Archives contestataires de Carouge.

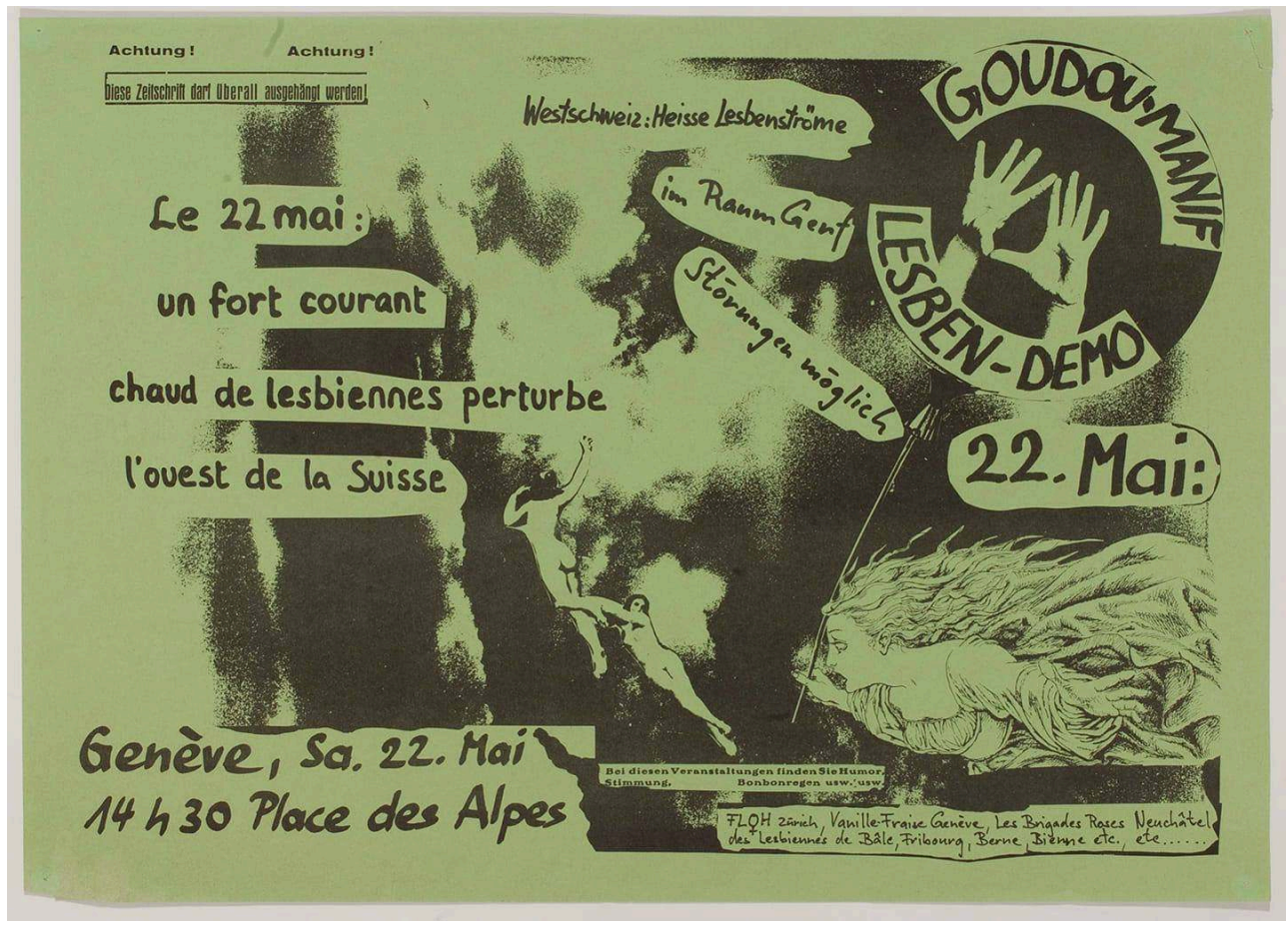

\section{Les émissions radiophoniques lesbiennes Sappho Revient et Canicule}

«Lesbiennes, c'est à vous qu'on s'adresse. Les autres peuvent écouter aussi, mais c'est à votre risque et péril...». Avec ces mots, s'ouvrait le 8 avril 1981 la première émission de 
radio pirate de Vanille-Fraise : "Sappho Revient », diffusée par la radio féministe Radio Pleine Lune. Depuis sa constitution, le groupe travaille pour créer ses propres lieux d'expression, avec la publication de son journal/fanzine Clit 007 et la diffusion de deux émissions radiophoniques faites "par et pour " des lesbiennes : "Sappho Revient " dans les années 1980 et «Canicule » dans les années $1990^{7}$. Le premier numéro de Clit 007 alertait les lectrices que « depuis l'automne 1980, il [existait] à Genève une radio de femmes-pirates qui [émettait] tous les 28 jours (cycle irrégulier) » et que « la technique [était] entièrement réalisée par des femmes $"^{8}$. Pour les femmes qui sont à l'origine du projet " la liberté des ondes est aussi importante que la liberté de la presse " (Joly, $1998: 82)$.

Dans les ondes de ces émissions, on pouvait écouter des discussions autour de la visibilité, la sexualité, la maternité, la culture et la politique lesbiennes; des actualités sur les luttes des femmes; des récits de voyage; des informations sur les conférences, les réunions, les camps d'été et les villages lesbiens à l'étranger; des extraits de livres ; des témoignages de lesbiennes helvétiques et internationales; ainsi que des musiques et chansons lesbiennes, dont le répertoire du fameux groupe allemand des Flying Lesbians.

L'initiative des émissions radio vient de Claire Sagnières, militante de Vanille Fraise que nous avons rencontrée en compagnie de Barbara Conrad lors d'un entretien collectif réalisé pendant un atelier. Voici un extrait de son témoignage: "Je venais d'avoir ma fille et je ne pouvais pas sortir, étant donné qu'il n'y avait personne qui pouvait la garder. J'ai donc pensé à une manière de continuer à militer depuis chez moi. C'est ainsi qu'est née l'idée de fabriquer des émissions radio avec les autres ».

Pour écouter les émissions, visitez l'archive en ligne créée par Claire Sagnières, qui a effectué la numérisation des cassettes originales qui se trouvent dans l'archive de Lestime.

Affiche de la radio de femmes La pleine lune @ Archives contestataires de Carouge.

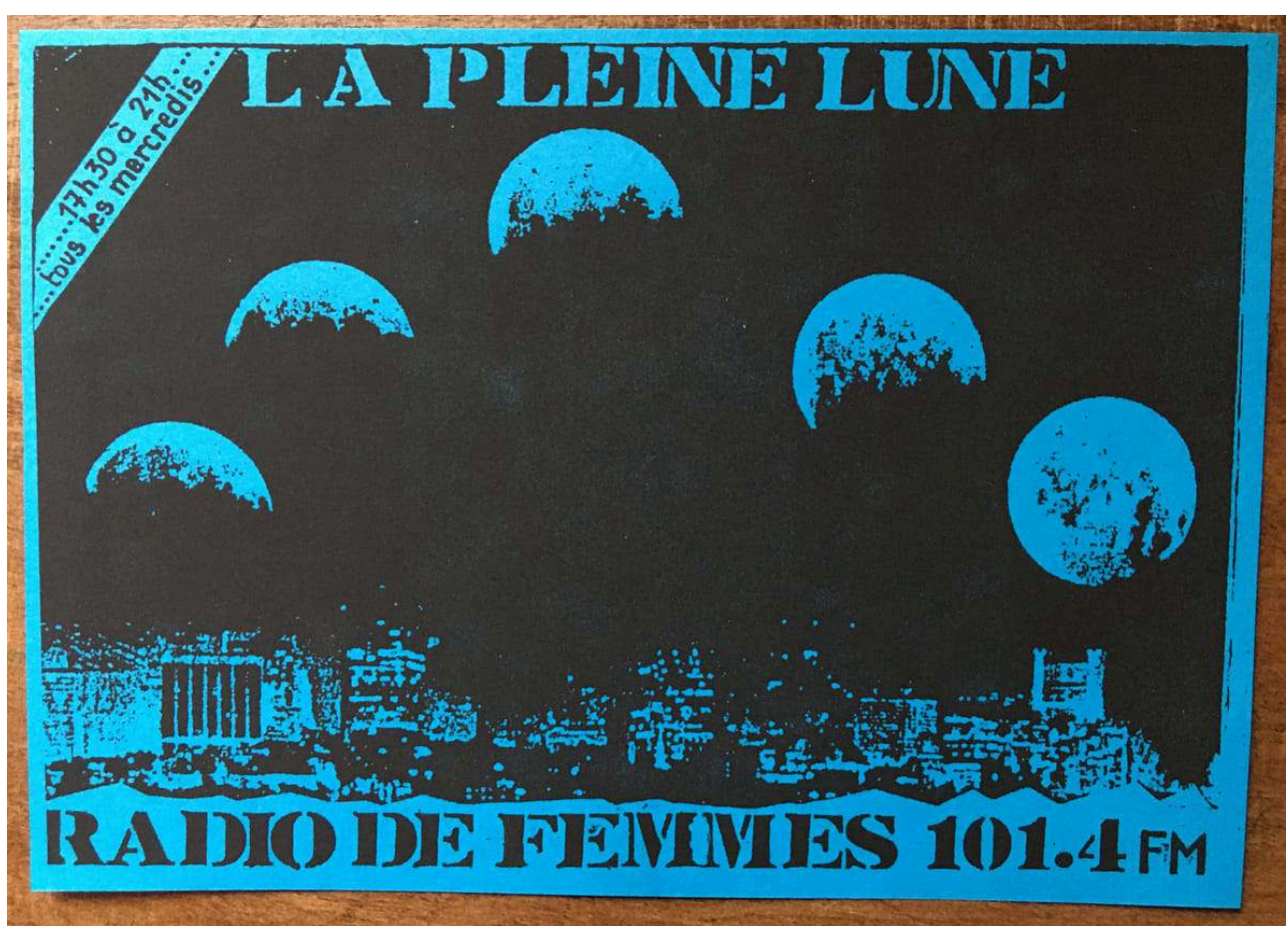

GLAD!, 11 | 2021 
Clit 007, n. 0, p. 7.

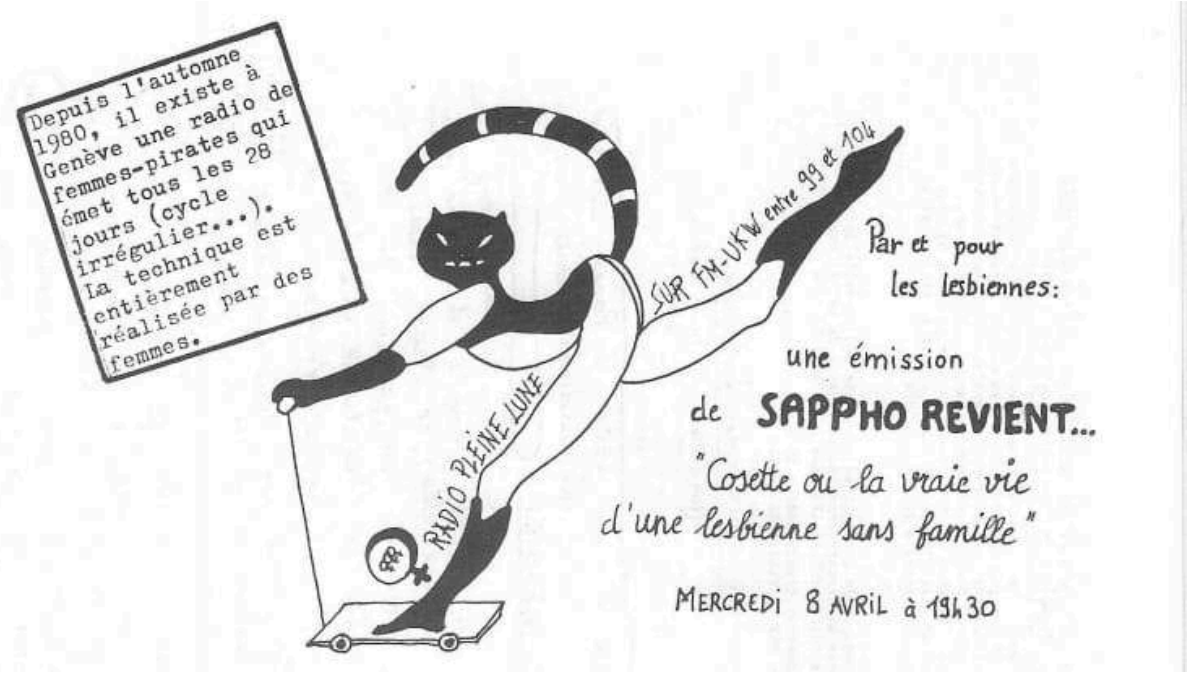

Logo de l'émission Canicule (1990-1992), source : https://clit007.ch/emissions-radios/.

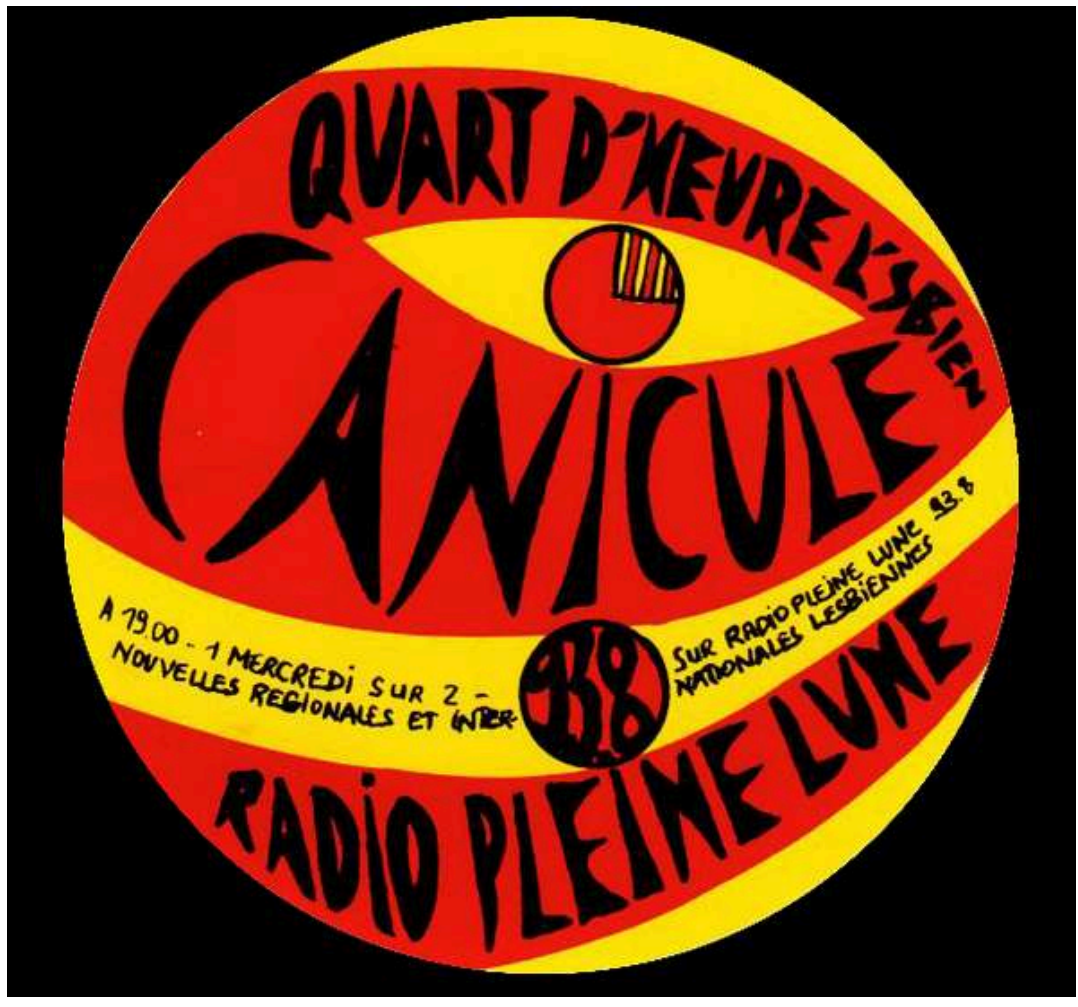

\section{La $8^{\mathrm{e}}$ conférence de l'ILIS à Genève}

Du 28 au 31 mars 1986 se tient à Genève la $8^{\text {e }}$ conférence de l'International Lesbian Information Service (ILIS), association née d'une scission au sein de l'International Lesbian, Gay, Bisexual, Trans and Intersex Association (ILGA) en 1980 pour favoriser l'organisation internationale et autonome des lesbiennes. La conférence est organisée par Vanille Fraise, qui prend en charge le secrétariat de l'ILIS de 1985 à 1986. Elle rassemble plus de 700 femmes venues d'une trentaine de pays d'Europe, Asie, Amérique latine, États-Unis 
et Afrique. L'organisation s'avère laborieuse: il faut répondre aux courriers, commander les billets d'avion, des visas, trouver des solutions d'hébergement, rechercher des traductrices, planifier les repas, préparer la conférence de presse et la manifestation de clôture. Par ailleurs, des collectes de fonds sont mises en place pour permettre la participation de lesbiennes issues de pays défavorisés. Durant la conférence, qui a lieu a Uni Dufour, sont prévus 16 ateliers théoriques, deux séances plénières, une fête, une manifestation, des stands de vente de livres. Par rapport aux autres conférences ILIS que l'ont précédée, cette $8^{\mathrm{e}}$ conférence s'engage à donner de la visibilité à des thématiques peu présentes dans les agendas des mouvements lesbiens occidentaux, comme la lutte contre le racisme, les enjeux postcoloniaux, la situation des lesbiennes $\mathrm{du}$ " tiers-monde » ainsi que la relation entre le racisme, l'antisémitisme et l'hétérosexisme. Certains des textes de présentation des ateliers sont republiés par Vanille Fraise dans le n.18 de mas 1986 du Clit 007 (désormais Bulletin de l'ILIS), dont un texte de Paola Bacchetta sur "Sexisme et racisme » traduit en anglais et en espagnol. $\mathrm{Au}$ tournant des années 1980, Genève devient donc un point névralgique dans l'articulation d'un mouvement lesbien transnational et intersectionnel qui s'ouvre à la réflexion sur les différences entre femmes.

En guise de clôture de la conférence, une manifestation pour les droits d'asile politique pour les lesbiennes de tous les pays est organisée le 29 mars 1986. Environ 500 femmes défilent dans les rues de Genève distribuant des tracts. Vanille Fraise adresse au HautCommissaire aux réfugiés (HCR) un long courrier qui demande d'élargir la notion de réfugié aux personnes persécutées en raison de leur orientation sexuelle. S'agissant d'une manifestation non autorisée, les trois organisatrices sont convoquées par la police. À la suite d'un procès, les trois femmes sont finalement acquittées.

Manifestation pour les droits d'asile pour les lesbiennes de tous les pays, 29 mars 1986, Archives de Lestime

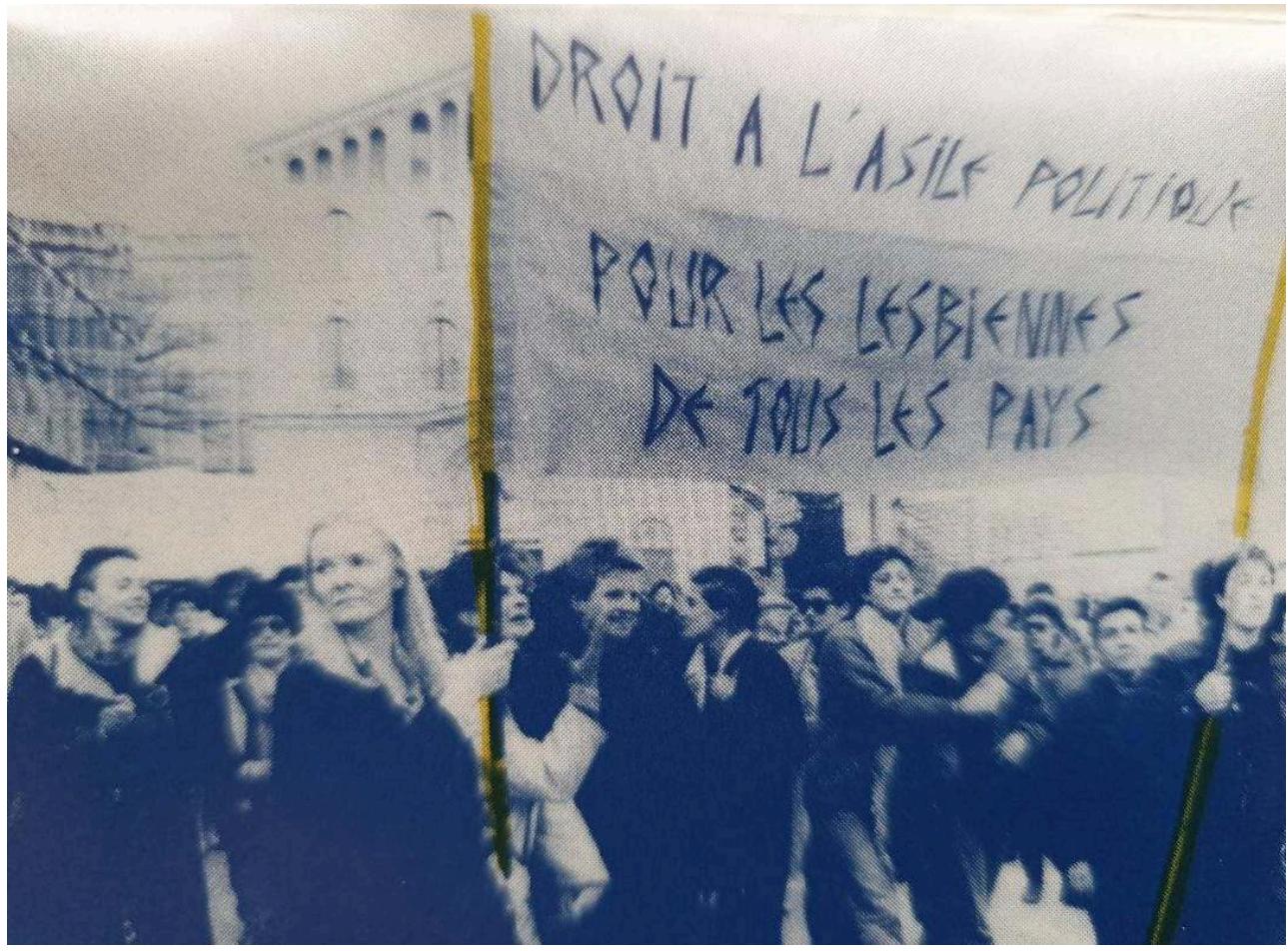




\section{Cafés, bars et boîtes de nuit}

En mars 1980, le magazine d'information Tell Quel consacre une émission aux cafés, boîtes de nuit et autres lieux de rencontre pour homosexuels qui commencent à se développer à Genève et en Suisse romande. Les lesbiennes n'y figurent pas. Cela tient au fait qu'elles souffrent d'une moindre visibilité dans l'espace public par rapport à la contrepartie masculine. Mais les lieux de rencontre lesbiens qui ont rassemblé des genevoises et des lesbiennes françaises ayant traversé la frontière pour le temps d'une soirée, ont été tout aussi nombreux à Genève, comme nous l'avons découvert en interviewant des femmes qui avaient l'habitude de les fréquenter. Les bars recouvrent une importance particulière dans l'histoire LGBTIQ+. Le "bar lesbien » notamment, comme le rappelle Emilie Martineau (2018), est un levier de visibilisation de la communauté lesbienne dans l'espace urbain.

A Genève, durant les années 1980 jusqu'au début des années 1990, les lesbiennes fréquentent le bar Chez Gaston au Square Pradier. Si les amis gays étaient acceptés, les hommes hétérosexuels ne sont pas les bienvenus. Le mobilier est composé de tables basses entourées de grands canapés. Gaston, une lesbienne butch qui avait connu le milieu parisien des années 1940, accueillit les clientes en costard-cravate et chemise blanche. Un autre lieu mythique des nuits lesbiennes genevoises est $L a$ Bretelle. Le local ouvre à la rue des Étuves 17 en 1979 grâce à l'accordéoniste Marie Claire Roulin et à son amie, qui rachètent le local du café à fondues la Channe Valaisanne. À la Bretelle, lesbiennes, gays, bi.e.s, trans* se fréquentent et rencontrent les habitué.e.s du quartier. Des travailleuses du sexe y font aussi escale. L'accordéon, le piano à bretelles d'où le bistrot tire son nom, occupe une place importante sur la scène. L'animation culturelle du lieu, qui devient rapidement très célèbre dans la ville comme le montre le reportage de la RTS Geneva gay map de 1979, est assurée par des activités très variées : soirées festives et dansantes, concerts, spectacles de théâtre, lectures de poésie. Sortir à la Bretelle représentait donc l'immersion dans une réalité non seulement festive, mais aussi militante. Un autre lieu genevois incontournable pour lesbiennes est le bar Chez Lilly. Situé au cœur du quartier populaire des Pâquis, il est resté ouvert de 1978 à 1990 grâce à Lilly, qui fut durant douze ans l'âme de ce lieu. Comme à l'époque on n'avait pas le droit d'interdire l'accès d'un bar aux hommes, Lilly, féministe convaincue, fait tout pour les démotiver afin qu'ils ne reviennent pas. Chez Lilly l'atmosphère était « cosy »: napperons glissés sous les verres, fleurs ornant les tables. Il régnait une ambiance plus proche du salon de thé que du bar de nuit. Les femmes y viennent avant tout pour discuter. Une légende urbaine racontée par nos interviewées prétend qu'une « guerre de style » opposait les filles plus chahuteuses de Chez Gaston et de la Bretelle à celles de chez Lilly. En 1996, trois amies: Chatty, Isabel et Christiane, tentent un club à sensibilité «femme », mais ouvert à tout le monde : Le Barbie. Elles choisissent l'espace d'une petite cave du squat de la rue Lissignol au Madone Bar. Le club va animer des soirées tous les vendredis soirs. Au début surtout lesbien, le public évoluera vers un mélange des genres qui rendra l'endroit différent à chaque fois. Plus tard, avec l'émergence du mouvement squat dans les années 1990, d'autres bars voient le jour à Genève. C'est le cas de Chez Brigitte (1994-2002), bar attenant au squat éponyme, le premier de Suisse romande à revendiquer son identité queer. L'association 360 et la première Marche des fiertés romandes du 5 juillet 1997 y voient le jour. En avril 1998, le bar Le Brigitte doit fermer ses portes à cause des attaques et des violences répétées provenant de l'extérieure. Laure, responsable du bar, raconte dans une émission de la 
RTS les raisons de la fermeture. Même si le bâtiment de la Rue Prévost-Martin n'existe plus, Chez Brigitte reste encore aujourd'hui un véritable monument. Dans le cadre de la Geneva Pride 2019, la Ville de Genève a soutenu une exposition-installation qui a proposé de redécouvrir l'histoire passionnante du premier squat LGBTIQ+ de Suisse.

Les soirées thématiques de Chez Brigitte brouillent les genres

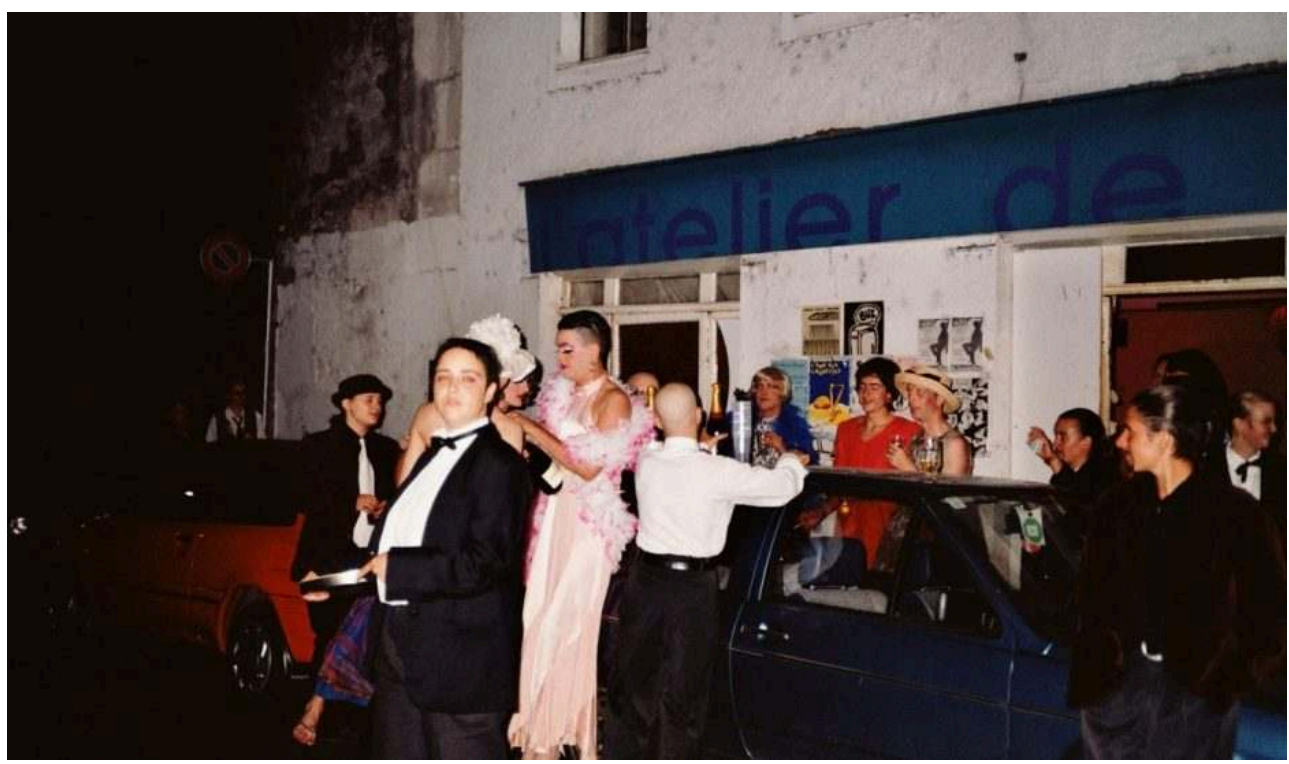

Source : «Chez Brigitte, havre queer_», Le Courrier, 26 juin 2019

Retrouvez l'histoire de l'ensemble de ces lieux via notre cartographie numérique.

\section{Du Centre femmes Natalie Barney à Lestime}

Les années 1988-1989 marquent le déménagement du Centre femmes du Boulevard Saint-Georges à l'Avenue Peschier dans le quartier de Champel. Les femmes qui reprennent le projet essayent de sortir d'une forme de clandestinité honteuse pour aller vers un lesbianisme plus assumé et joyeux. Fête et visibilité sont les nouveaux mots-clés. Elles réalisent qu'elles se trouvent à un tournant générationnel et politique : d'une part, elles doivent assurer la continuité avec les « féministes historiques » issues des luttes des années 1970, qui tenaient à la non-mixité du lieu ; de l'autre, elles doivent faire face à celles qui voulaient un rapprochement avec le mouvement gay. Ce clivage devient récurrent lors des réunions et des assemblées et parfois surgissent des conflits. Les demandes de subventions auprès du Conseil municipal remplacent l'idée initiale d'un autofinancement, devenu impossible à cause du loyer élevé. Suite à des négociations, les autorités octroient une subvention, mais refusent toutefois l'appellation de "centre lesbien » proposée par les militantes. Le nom choisi sera celui de Centre femmes Natalie Barney, en hommage à la fameuse salonnière parisienne d'origine américaine qui était ouvertement lesbienne. Les militantes mettent sur pied un centre de documentation, un bar et organisent régulièrement des soirées dansantes, des diners, des grillades dans le jardin, des expositions artistiques, des weekends à l'étranger. Elles veillent à la coexistence des pôles politique et convivial, même si les tensions entre les «politiques » et les «festives " ne manquent pas. Elles publient un journal de réflexion autour du lesbianisme qui parait une seule fois: Gouts doux, et 
organisent deux groupes d'écoute: un sur la maternité lesbienne et un sur les difficultés du coming-out. De par ses liens à l'international, la publicité du centre Natalie Barney apparait dans des journaux lesbiens américains. Il s'agit d'un moment heureux pour la communauté lesbienne genevoise, qui pour autant ne peut pas oublier les évènements dramatiques liés au sida et à la parte d'ami.e.s qui ont également tissé la trame de cette époque.

Entre la fin des années 1980 et le début des années 2000, deux déménagements importants interviennent. Le 30 novembre 2002, l'association se rebaptise Lestime et inaugure ses nouveaux locaux au 5 rue de l'Industrie dans le quartier des Grottes. Pour célébrer l'événement, Catherine Gaillard, sa première présidente et une des premières figures politiques de Suisse romande à faire son coming-out, sollicite la vidéaste valaisanne Carole Roussopoulos pour réaliser un documentaire sur l'association intitulé «Qui a peur des amazones?» (2003, $27 \mathrm{~min})$. Avec l'accord de Lestime, la RTS a publié un extrait de ce documentaire original sur son site d'Archives. Désormais durablement subventionnée, Lestime continue ses activités dans la poursuite des objectifs des mouvements féministes et lesbiens du passé sans oublier les nouveaux défis d'inclusivité du présent. Le travail sur la mémoire et les archives, ainsi que les enjeux de transmission intergénérationnelle, demeurent des priorités dans ses actions de visibilité.

Pour en connaitre davantage sur cette histoire, vous pouvez consulter la brochure éditée en 2007 à l'occasion des 5 ans de Lestime et des 35 ans du mouvement lesbien genevois, disponible en ligne via notre frise chronologique. Ou encore, la brochure en ligne Genève, fière de son histoire LGBTIQ+ éditée en 2021 par la Ville de Genève, qui retrace quelques faits marquants de l'histoire LGBTIQ+ à Genève depuis les années 1970 à travers une sélection de photos et de documents d'archives rassemblés grâce à la mobilisation du réseau associatif.

Nous continuerons dans les prochains mois et années à explorer des dispositifs coopératifs et participatifs ainsi que des médiations numériques afin de rendre visible notre « herstory ». Suivez-nous sur les réseaux sociaux (facebook et instagram) pour connaitre nos actualités et y participer ! 
Logo du Centre femme Nathalie Barney dessiné par Kiki, Archives de Lestime.

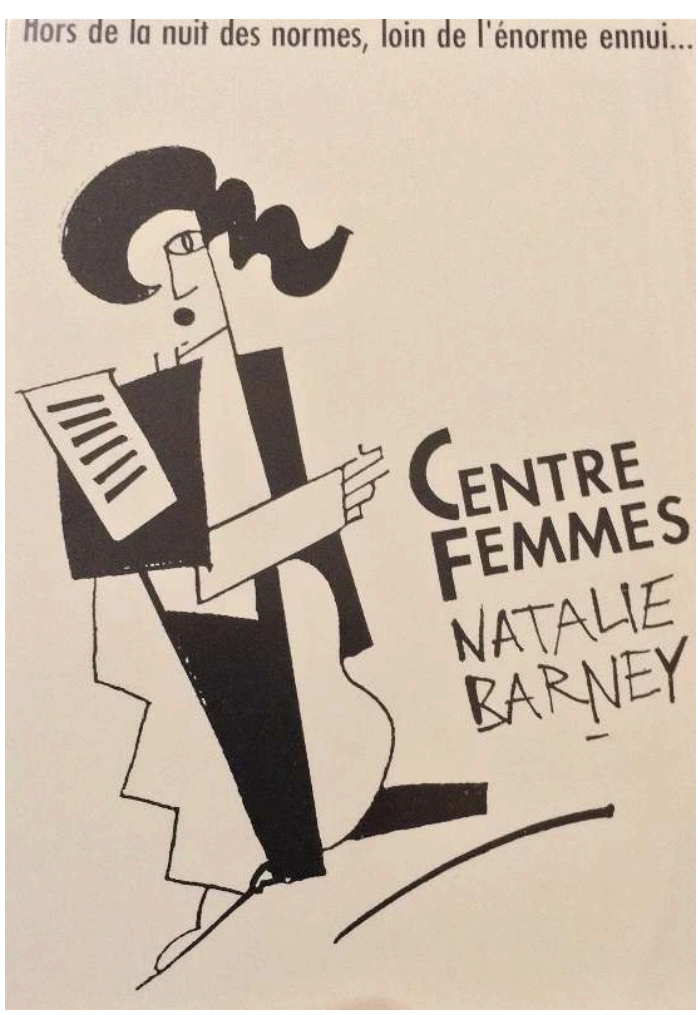

\section{BIBLIOGRAPHIE}

ASTIER, Lorraine, MATRAS, Mathilde, TOPINI, Carolina. 2019. «Étude exploratoire des archives des luttes LGBTIQ+ à Genève (1970-2000). État des lieux et propositions de solutions » Rapport de recherche sous mandat de l'Agenda 21, Ville de Genève, [en ligne] URL : https://www.geneve.ch/sites/ default/files/2021-06/rapport-archivesLGBTIQ2019-ville-de-geneve.pdf

ASTIER, Lorraine, MATRAS, Mathilde, TOPINI, Carolina. 2020. «Quelle place pour les archives des mouvements LGBTIQ+ ? Une réflexion genevoise » Arbido, Revue professionnelle suisse pour les archives, bibliothèques et centres de documentation 2020(2) [en ligne] URL : https://www.geneve.ch/ sites/default/files/2021-06/rapport-archivesLGBTIQ2019-ville-de-geneve.pdf

BONNET, Marie-Jo. 1998. « De l'émancipation amoureuse des femmes dans la cité » Les Temps modernes $598: 85-112$.

CAMERONI, Martine et MEYER, Sophie. s.d. « Notre histoire compte » Archives en ligne de la RTS [en ligne] URL : https://www.rts.ch/archives/grands-formats/10534402--notre-histoirecompte-.html.

CHAMBERLAND, Line. 1993. « Remembering Lesbian Bars: Montréal, 1955-1975 » Journal of Homosexuality 25(3) : 231-270. 
DARDEL, Julie. 2007. Révolution sexuelle et Mouvement de Libération des Femmes à Genève, 1970-1977. Lausanne : Antipodes.

DAVIS, Madeline et LAPOVSKY KENNEDY, Elizabeth. 1986. « Oral History and the Study of Sexuality in the Lesbian Community: Buffalo, New York, 1940-1960 » Feminist Studies 12(1) : 7-26.

EICHHORN, Kate. 2013. The Archival Turn in Feminism: Outrage in Order. Philadelphie : Temple University Press.

ENKE, Anne. 2007. Finding the Movement: Sexuality, Contested Space, and Feminist Activism. Durham : Duke University Press.

Front des lesbiennes radicales (ed.). 2010. Textes de la rencontre de juin 1982 [en ligne] URL : https:// fmsh.bach.anaphore.org/archives/show/FR075FMSH_000000067_de-292

HOGAN, Kristen. 2016. The feminist bookstore movement: lesbian antiracism and feminist accountability. Durham : Duke University Press Books,

IDIER, Antoine. 2018. « Absences, silences et politique de l'histoire ", in Archives des mouvements LGBT+ : Une histoire des luttes de 1890 à nos jours, IDIER, Antoine (ed). Paris : Éditions Textuel.

JOLY, Helene. 1998. « De Sappho s'en fout à Vanille fraise (1972-1986) : Histoire du mouvement lesbien genevois, ", Mémoire de Master, Études genre, Université de Genève.

MARTINEAU, Émilie. 2018. « Le bar lesbien, un commerce militant en voie de réapparition à Bruxelles » Cahiers du Master Genre 2018.

MEYER, Sophie. 2010. «Présentation de soi et représentation de l'autre : l'image des femmes homosexuelles dans les émissions de la Télévision Suisse Romande entre 1954 et 2009 ", Mémoire en Etudes de Genre, Université de Genève.

OLLAGNIER, Edmee et BUDRY, Maryelle (dir.). 1999. Mais qu'est-ce qu'elles voulaient ? Histoires de vie du MLF à Genève. Lausanne : Éditions d'en bas.

SENTIS, Isabelle et SALEM, Diego. 2019. « Célébrons les riotts de Stonewall en documentant nousmême l'histoire de nos bars lesbiens ", Lesbian Lives Conference, University of Brighton, UK, 15-16 March 2019, The Politics of (In)Visibility.

SCHULMAN, Sarah. 2018. La Gentrification des esprits. Paris : B42.

\section{ANNEXES}

https://www.lestime.ch

https://www.queercode.net/projets

http://constellationsbrisees.net/cartographie-notre-histoire-compte.html

https://www.rts.ch/archives/

https://www.geneve.ch/fr/themes/developpement-durable/municipalite/ engagements-societe/egalite-diversite/orientation-sexuelle-identite-genre/memoireslgbtiq 


\section{NOTES}

1. Cf. «Une interview de Joan Nestle et de Deborah Edel », p. 63, in Front des lesbiennes radicales (ed.), Textes de la rencontre de juin 1982, réédités en 2010 sous forme de brochure.

2. Lestime_est une association lesbienne et féministe ouverte aux femmes cis et trans*, bisexuelles et queer. Espace communautaire, culturel et politique, elle a été créée à Genève en 2002 et a succédé à de nombreuses associations féministes dont le Centre Femmes au boulevard Saint-Georges (1976), puis La Maison à Champel, rebaptisée Centre Femmes Natalie Barney (CFNB).

3. Queer Code est un espace numérique collaboratif pour rendre visibles les parcours de vie des femmes ayant aimé des femmes, qu'elles furent cisgenres ou transgenres, durant la Seconde Guerre Mondiale, leurs résistances, leurs émancipations et leurs amours.

4. Théorisée, entre autres, par le Collectif Archives LGBTQI de Paris.

5. Lorraine Astier, Mathilde Matras, Carolina Topini, Étude exploratoire des archives des luttes LGBTIQ+ à Genève (1970-2000). État des lieux et propositions de solutions, rapport de recherche sous mandat de l'Agenda 21-Ville de Genève, février 2019, pp. 86. Cf. également des mêmes autrices l'article « Quelle place pour les archives des mouvements LGBTIQ+ ? Une réflexion genevoise », in Arbido. Revue professionnelle suisse pour les archives, bibliothèques et centres de documentation, numéro sur « Représentations et mises en scènes de la société », 2020/2, disponible en ligne.

6. Des références aux parcours des lesbiennes féministes au sein du MLF-Genève sont également mentionnées dans deux ouvrages : Edmee Ollagnier et Maryelle Budry eds., Mais qu'est-ce qu'elles voulaient? Histoires de vie du MLF à Genève, Lausanne, Éditions d'en bas, 1999 ; et Dardel Julie, Révolution sexuelle et Mouvement de Libération des Femmes à Genève, 1970-1977, Antipodes, 2007.

7. Un projet de catalogage et numérisation des archives sonores de la Radio Plaine Lune est actuellement en cours au sein des Archives contestataires de Carouge. Sur leur site, il est disponible un début d'inventaire.

8. Clit 007 n. 0, p. 7.

\section{RÉSUMÉS}

L'article présente la démarche et les enjeux du projet associatif «Nos lieux, nos fêtes, nos combats : notre histoire compte ". Dédié à la collecte et à la transmission d'archives relatives à l'histoire des mouvements féministes et lesbiens de Genève, le projet vise à produire un espace d'apprentissage collectif. La contribution crée un paysage visuel et sonore pour accompagner la découverte de quelques moments marquants de cette histoire méconnue: les actions contestataires du MLF-Genève ; les fameux « bals des chattes sauvages »; la naissance de la revue Clit 007 (1980-1986) ; la « Goudou-Manif » de 1982 ; les émissions radiophoniques Sappho Revient et Canicule (années 1980-1990) ; la 8e conférence de l'ILIS à Genève de 1986 ; les cafés, bars et boîtes de nuit ; la création du Centre femmes Natalie Barney et de Lestime.

This article presents the approach and the stakes involved in the activist project «Our places, our parties, our fights: our history matters ». Dedicated to the collection and transmission of archives regarding the history of feminist and lesbian movements in Geneva, this project aims to provide a space for collective learning. The contribution creates a visual journey and a soundscape to introduce the reader to some pivotal moments of this little-known history: the 
provocative actions of the WLM-Geneva; the famous " bals des chattes sauvages "; the launch of the lesbian magazine Clit 007 (1980-1986); the 1982 «Goudou-Manif»; the radio broadcasts Sappho Revient and Canicule (1980s-1990s); the 8th ILIS conference in Geneva in 1986; the cafes, bars and nightclubs; the creation of the Natalie Barney Women's Center and Lestime.

\section{INDEX}

Mots-clés : archives, lesbiennes, féminisme, histoire, LGBTQ, militantisme, mouvements sociaux, cartographie numérique

\section{AUTEURS}

\section{CAROLINA TOPINI}

Assistante-doctorante à l'Institut des Études genre de l'Université de Genève, où elle enseigne les théories et les mouvements féministes et réalise une thèse sur « Espaces, rencontres, voyages, traductions. La fabrique d'un féminisme transnational dans les années 1970-1990 (Italie, Europe, États-Unis) ». Dans son travail elle s'intéresse aux circulations transnationales des idées et des pratiques féministes ; aux théories féministes, queer et post/décoloniales ; à l'histoire et l'actualité des mouvements féministes et LGBTIQ+ ainsi qu'aux théories et épistémologies des archives. Elle est membre du collectif Notre histoire compte et du collectif de traduction transféministe Onna Pas.

\section{ISABELLE SALEM DIEGO SENTIS}

Performeuse, art-thérapeute et chercheuse impliquée dans des projets articulant santé, culture, archives et visibilité auprès de différentes associations féministes et LGBTQI*. Iel a cofondé le collectif Queercode et est membre du collectif Notre histoire compte. Iel est intervenu.e entre autres à Paris en 2016 lors du Colloque sur les Archives lesbiennes, en 2016 à l'Université de Londres lors de la rencontre internationale Archives, bibliothèques, musées et collections LGBTQ; à l'Université de Liverpool en 2018 et à celle de Brighton en 2019 sur les archives lesbiennes ; en 2017 au Colloque des Gays Games au Ministère de la santé à Paris et à l'Université de Concordia à Montréal en 2018 sur les connexions entre archives et santé LBT, ou encore au Mucem de 2018 à 2021sur les archives de la lutte contre le Sida. 